\title{
Direct Adaptive Dynamic Compensation for Minimum Phase Systems With Unknown Relative Degree
}

\author{
Jesse B. Hoagg and Dennis S. Bernstein
}

\begin{abstract}
We consider parameter-monotonic direct adaptive control for single-input-single-output minimum-phase linear time-invariant systems with knowledge of the sign of the high-frequency gain (first nonzero Markov parameter) and an upper bound on the magnitude of the high-frequency gain. The first part of the paper is devoted to fixed-gain analysis of single-parameter high-gain-stabilizing controllers. Two novel fixed-gain dynamic compensators are presented for stabilizing minimum-phase systems. One compensator stabilizes systems with arbitrary-but-known relative degree, while the other utilizes a Fibonacci series construction to stabilize systems with unknown-but-bounded relative degree. Next, we provide a general treatment of parameter-monotonic adaptive control, including a result that guarantees state convergence to zero. This result is then combined with the high-gain-stabilizing controllers to yield parameter-monotonic direct adaptive dynamic compensation for minimum-phase systems with either arbitrary-but-known or unknown-but-bounded relative degree.
\end{abstract}

Index Terms-Adaptive control, Fibonacci, parameter monotonic, relative degree.

\section{INTRODUCTION}

$\mathbf{M}$ ANY adaptive control methods rely on parameter estimation algorithms such as recursive least squares, gradient descent, and projection algorithms [1], [2]. Parameter estimation algorithms are used with indirect adaptive control methods to identify plant parameters, and with direct adaptive control methods to update controller parameters. Parameter-estimation-based adaptive controllers generally require an increasing number of adaptive parameters as the order of the system increases. For example, the dimension of a direct model reference adaptive controller increases at least linearly with the order of the plant, while the dimension of a recursive-least-squares-based algorithm increases quadratically with the order of the plant.

Alternatively, high-gain adaptive stabilization methods typically invoke a minimum phase assumption since zeros attract poles under high gain [3]-[12]. Adaptive high-gain proportional feedback can stabilize square multi-input multi-output systems that are minimum phase and relative degree one [3], [5], [6]. Adaptive controllers that do not require knowledge of the sign of the high-frequency gain are called universal stabilizers [6],

Manuscript received September 9, 2004; revised May 3, 2005, June 20, 2005, and May 1, 2006. Recommended by Associate Editor M. Demetriou.

The authors are with the Aerospace Engineering Department, the University of Michigan, Ann Arbor, MI 48109-2140 USA (e-mail: dsbaero@umich.edu).

Color versions of one or more of the figures in this paper are available online at http://ieeexplore.ieee.org.

Digital Object Identifier 10.1109/TAC.2007.894512
[13]-[18]. However, these controllers usually do not perform well on high-order systems due to slow convergence of the gainsearching algorithms.

In [7], high-gain dynamic compensation is used to guarantee output convergence of single-input-single-output (SISO) minimum phase systems with arbitrary-but-known relative degree. This work is surprising since classical root locus is not high-gain stable for plants with relative degree exceeding two. The dynamic compensators in [7] are of order $1 / 2\left(r^{2}-r\right)$, where $r$ is the relative degree. However, as we show in Section II, the results of [7] can fail when the relative degree of the plant is greater than four. Related work on high-gain adaptive control of systems with arbitrary-but-known relative degree includes [19], [20], which use high-gain observers.

In this paper, we first develop a class of high-gain dynamic compensators that use knowledge of the sign of the high-frequency gain to stabilize SISO minimum-phase systems with arbitrary-but-known relative degree. These controllers are of lower order than those in [7]. Furthermore, we construct a novel class of high-gain dynamic compensators for SISO minimum-phase systems with unknown-but-bounded relative degree. This construction uses the Fibonacci series and a variation of root locus.

The present paper also includes a general treatment of parameter-monotonic adaptive control. In [3], [5], and [6], the stability proofs for high-gain adaptive systems require that the control signal be linear in the adaptive parameter and the closed-loop system be affine in the adaptive parameter. Although the control signal in [7] is not linear in the adaptive parameter, the proof of output convergence requires that the adaptive parameter be a uniformly continuous function of time. This a priori assumption on the adaptive parameter is equivalent to assuming that the output is bounded. In the present paper, a parameter-monotonic adaptation law is shown to guarantee state convergence to zero for a large class of high-gain-stable closed-loop systems. The parameter-monotonic adaptive law incorporates an exponentially decaying factor, which has no counterpart in [3] and [5]-[7].

Finally, the parameter-monotonic adaptive law is combined with the Fibonacci-based high-gain dynamic compensator. Thus, the main result of the paper is parameter-monotonic adaptive stabilization of SISO minimum-phase systems with unknown-but-bounded relative degree.

In Section II, we present a motivating counterexample to the results presented in [7]. In Section III, we introduce the notion of parameter-dependent dynamic compensation. Section IV summarizes relevant root locus results, including a proportional feedback controller for systems with relative degree not exceeding two. In Section V, we analyze a fixed-gain compensator 


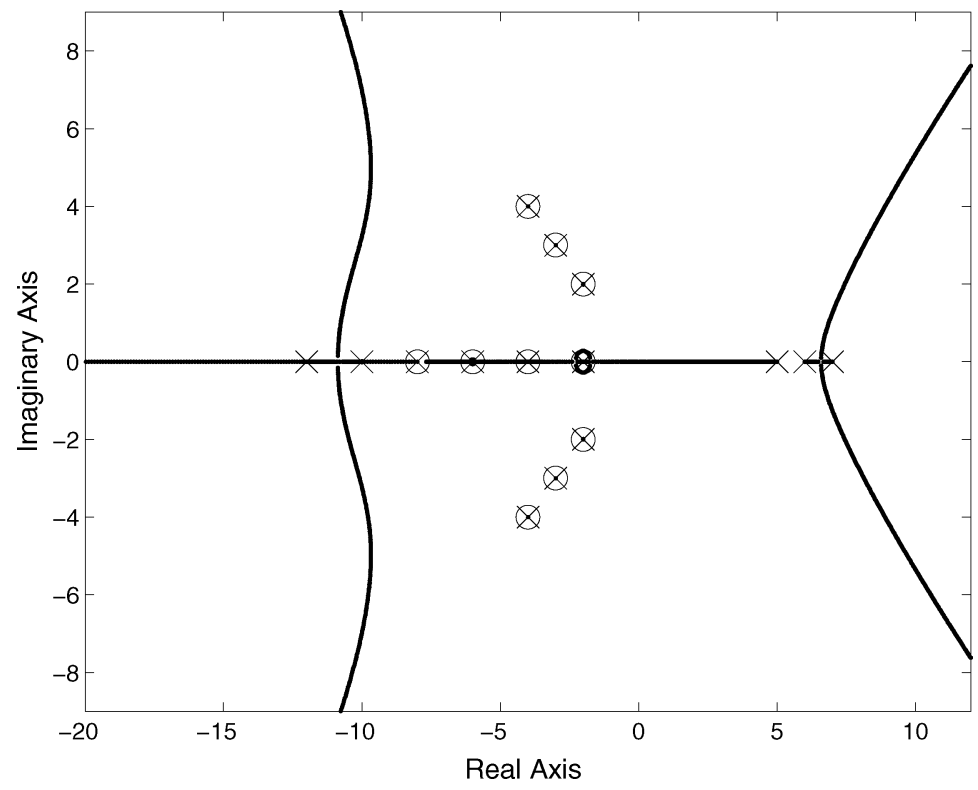

Fig. 1. Root locus for the closed-loop dynamics of the controller proposed in [7]. The system is not high-gain stable.

that stabilizes minimum-phase systems of arbitrary-but-known relative degree. Section VI presents a fixed-gain analysis of a Fibonacci-based compensator that stabilizes systems with unknown-but-bounded relative degree. In Section VII, we present parameter-monotonic adaptive stabilization. Section VIII presents parameter-monotonic adaptive stabilization for minimum-phase systems with unknown-but-bounded relative degree. Numerical examples are given in Sections IX and $\mathrm{X}$. Conclusions are given in Section XI.

\section{Counterexample to the Results of [7]}

Consider the unstable plant

$$
G(s)=\frac{q(s)}{p(s)}=\frac{1}{(s-5)(s-6)(s-7)(s+10)(s+12)} .
$$

Lemma 4 and [7, Fig. 1] propose a 10th-order controller to highgain stabilize (2.1). To satisfy the hypotheses of Lemma 4, an upper bound on the high-frequency gain of the plant is chosen to be $g_{0}=2.5$. The gains $g_{1}=2, g_{2}=5, g_{3}=3$, and $g_{4}=2$ are chosen so that the polynomial $g(s) \triangleq s^{5}+g_{4} s^{4}+$ $g_{3} s^{3}+g_{2} s^{2}+g_{1} s+g_{0}$ is Hurwitz. Furthermore, define the monic Hurwitz polynomials $r_{1}(s) \triangleq s^{4}+10 s^{3}+40 s^{2}+80 s+64$, $r_{2}(s) \triangleq s^{3}+12 s^{2}+54 s+108, r_{3}(s) \triangleq s^{2}+8 s+32$, and $r_{4}(s) \triangleq s+8$. The controller given in [7, Lemma 4] yields the closed-loop characteristic polynomial

$$
\begin{aligned}
\tilde{p}(s) \triangleq & p(s) \prod_{i=1}^{4} r_{i}(s)+k g_{4} p(s) \prod_{i=1}^{3} r_{i}(s) \\
& +k^{2} g_{3} p(s) \prod_{\substack{i \neq 3 \\
i=1}}^{4} r_{i}(s)+k^{3} g_{2} p(s) \prod_{\substack{i \neq 2 \\
i=1}}^{4} r_{i}(s) \\
& +k^{4} g_{1} p(s) \prod_{i=2}^{4} r_{i}(s)+k^{5} q(s) \prod_{i=1}^{4} r_{i}(s) .
\end{aligned}
$$

Fig. 1 provides a root locus for $\tilde{p}(s)$ as $k \rightarrow \infty$. The zero-gain $(k=0)$ pole locations are shown by $\times$ 's, while the locations of the roots of $r_{1}(s), \ldots, r_{4}(s)$, which attract certain poles, are shown by o's. Ten of the closed-loop poles converge to the stable roots of $r_{1}(s), \ldots, r_{4}(s)$ as $k \rightarrow \infty$. The real parts of three of the remaining five closed-loop poles approach minus infinity as $k \rightarrow \infty$. However, the real parts of the two remaining poles approach plus infinity as $k \rightarrow \infty$. Thus, the closed-loop system is not stable for all sufficiently large $k$. However, [7, Lemma 4] claims that there exists $k_{\mathrm{S}}>0$ such that, for all $k>k_{\mathrm{S}}$, $\tilde{p}(s)$ is asymptotically stable. In fact, the controller proposed in [7] with parameters $g_{0}=2.5, g_{1}=2, g_{2}=5, g_{3}=3$, and $g_{4}=2$ fails to stabilize all unstable relative-degree- 5 plants with a high-frequency gain of 1 .

The error in [7] can be traced to the application of Lemma 3 to obtain Lemma 4. The Hurwitz hypothesis on $g(s)$ is not sufficient for stability of the closed-loop system. However, it suffices to require that $s^{5}+g_{4} s^{4}+g_{3} s^{3}+g_{2} s^{2}+g_{1} s+\eta g_{0}$ be Hurwitz for all $\eta \in(0,1]$. The polynomial $g(s)$ with $g_{0}=2.5$, $g_{1}=2, g_{2}=5, g_{3}=3$, and $g_{4}=2$ does not possess this property. We revisit this example in Section IX.

\section{PARAmeter-DePendent Dynamic Compensation}

We consider the strictly proper SISO linear time-invariant system

$$
y=G(s) u
$$

where

$$
G(s) \triangleq \delta \beta \frac{z(s)}{p(s)} .
$$

We make the following assumptions.

(A1) $z(s)$ is a real monic Hurwitz polynomial but is otherwise unknown.

(A2) $p(s)$ is a real monic polynomial but is otherwise unknown. 
(A3) $z(s)$ and $p(s)$ are coprime.

(A4) The magnitude $\beta$ of the high-frequency gain satisfies $0<\beta \leq b_{0}$, where $b_{0} \in \mathbb{R}$ is known.

(A5) The $\operatorname{sign} \delta= \pm 1$ of the high-frequency gain is known. For later use, we define the notation $m \triangleq \operatorname{deg} z(s), n \triangleq$ $\operatorname{deg} p(s)$, and $r \triangleq n-m$.

Let $z_{k}(s)$ and $p_{k}(s)$ be parameter-dependent polynomials, that is, polynomials in $s$ over the reals whose coefficients are functions of a real parameter $k$. Furthermore, define the parameter-dependent transfer function $G_{k}(s) \triangleq z_{k}(s) / p_{k}(s)$, where, for all $k \in \mathbb{R}, p_{k}(\cdot) \not \equiv 0$. Note that the polynomials $z_{k}(s)$ and $p_{k}(s)$ need not be coprime for all $k \in \mathbb{R}$.

Definition 3.1: $p_{k}(s)$ is high-gain Hurwitz if there exists $k_{\mathrm{s}}>$ 0 such that $p_{k}(s)$ is Hurwitz for all $k \geq k_{\mathrm{s}}$.

Definition 3.2: $G_{k}(s)$ is high-gain stable if there exist parameter-dependent polynomials $z_{k}(s)$ and $p_{k}(s)$ such that $p_{k}(s)$ is high-gain Hurwitz and, for all $k \in \mathbb{R}, G_{k}(s)=z_{k}(s) / p_{k}(s)$.

Now, consider the system (3.1) and (3.2) with the input $u=$ $u_{0}-u_{c}$, where $u_{c}$ is given by

$$
u_{c}=\hat{G}_{k}(s) y
$$

where $\hat{G}_{k}(s) \triangleq \hat{z}_{k}(s) / \hat{p}_{k}(s)$ and $\hat{z}_{k}(s)$ and $\hat{p}_{k}(s)$ are parameter-dependent polynomials. For example, letting $\hat{z}_{k}(s)=\delta k$ and $\hat{p}_{k}(s)=1$ yields $\hat{G}_{k}(s)=\delta k$, and the closed-loop poles can be determined by classical root locus. In general, the closedloop transfer function from input $u_{0}$ to output $y$ is

$$
\tilde{G}_{k}(s) \triangleq \frac{G(s)}{1+\hat{G}_{k}(s) G(s)}=\frac{\tilde{z}_{k}(s)}{\tilde{p}_{k}(s)}
$$

where $\tilde{z}_{k}(s) \triangleq \delta \beta z(s) \hat{p}_{k}(s)$ and $\tilde{p}_{k}(s) \triangleq p(s) \hat{p}_{k}(s)+$ $\delta \beta z(s) \hat{z}_{k}(s) . \tilde{G}_{k}(s)$ is high-gain stable if its parameter-dependent characteristic polynomial $\tilde{p}_{k}(s)$ is high-gain Hurwitz.

\section{Static Feedback for Relative DegReE $r=1$ AND $r=2$}

In this section, we stabilize (3.1) and (3.2) with proportional feedback $u_{c}=\delta k y$ so that

$$
\tilde{G}_{k}(s) \triangleq \frac{G(s)}{1+\delta k G(s)}=\frac{\tilde{z}(s)}{\tilde{p}_{k}(s)}
$$

where $\tilde{z}(s) \triangleq \delta \beta z(s)$ and $\tilde{p}_{k}(s) \triangleq p(s)+k \beta z(s)$. The following results from root locus analysis [21], [22] are needed.

Lemma 4.1: Let $q(s)$ be a degree $\nu \geq 1$ monic polynomial, and let $v(s)$ be a degree $\nu-1$ polynomial with positive leading coefficient. Then, as $k \rightarrow \infty, \nu-1$ roots of $q(s)+$ $k v(s)$ converge to the roots of $v(s)$, and the remaining root approaches $-\infty$. If, in addition, $v(s)$ is Hurwitz, then $q(s)+k v(s)$ is high-gain Hurwitz.

Lemma 4.2: Let $q(s)$ be a degree $\nu \geq 2$ monic polynomial, and let $v(s)$ be a degree $\nu-2$ polynomial with positive leading coefficient. Let $\lambda_{1}(q), \ldots, \lambda_{\nu}(q)$ and $\lambda_{1}(v), \ldots, \lambda_{\nu-2}(v)$ denote the roots of $q(s)$ and $v(s)$, respectively, and define the root locus center

$$
\xi(q, v) \triangleq \frac{1}{2}\left(\sum_{i=1}^{\nu} \lambda_{i}(q)-\sum_{j=1}^{\nu-2} \lambda_{j}(v)\right) .
$$

Then, as $k \rightarrow \infty, \nu-2$ roots of $q(s)+k v(s)$ converge to the roots of $v(s)$, and the two remaining roots approach $\xi(q, v) \pm$ $\jmath \infty$. If, in addition, $v(s)$ is Hurwitz and $\xi(q, v)<0$, then $q(s)+$ $k v(s)$ is high-gain Hurwitz.

The following consequences of lemmas 4.1 and 4.2 do not require knowledge of $b_{0}$ in (A4).

Proposition 4.1: Consider the closed-loop transfer function (4.1). If $r=1$ or if $r=2$ and $\xi(p, z)<0$, then $\tilde{p}_{k}(s)$ is highgain Hurwitz, and thus $\tilde{G}_{k}(s)$ is high-gain stable. Furthermore, as $k \rightarrow \infty$, $m$ of the roots of $\tilde{p}_{k}(s)$ converge to the roots of $z(s)$. If $r=1$, then the remaining root approaches $-\infty$. If $r=2$, then the remaining two roots approach $\xi(p, z) \pm \jmath \infty$.

Proposition 4.2: Consider the closed-loop transfer function (4.1). If $r=2$ and $\xi(p, z)>0$ or if $r \geq 3$, then $\tilde{G}_{k}(s)$ is not high-gain stable. In particular, if $r=2$, then, as $k \rightarrow \infty$, two roots of $\tilde{p}_{k}(s)$ approach $\xi(p, z) \pm \jmath \infty$. If $r \geq 3$, then, as $k \rightarrow \infty$, the real part of at least one root of $\tilde{p}_{k}(s)$ approaches $+\infty$.

\section{DYNAMIC COMPENSATION FOR SYSTEMS With ARBITRARY-BUT-KNOWN RELATIVE DEGREE}

Consider the feedback (3.3) with the strictly proper controller

$$
\hat{G}_{k}(s) \triangleq \frac{\delta k^{r+1} \hat{z}(s)}{s^{r}+k b_{r} s^{r-1}+\cdots+k^{r-1} b_{2} s+k^{r} b_{1}}
$$

where $k, b_{1}, \ldots, b_{r}$ are real and $\hat{z}(s)$ is a degree $r-1$ monic polynomial. Then

$$
\tilde{G}_{k}(s) \triangleq \frac{G(s)}{1+\hat{G}_{k}(s) G(s)}=\frac{\tilde{z}_{k}(s)}{\tilde{p}_{k}(s)}
$$

where

$$
\begin{aligned}
\tilde{z}_{k}(s) \triangleq & \delta \beta z(s)\left[s^{r}+k b_{r} s^{r-1}+\cdots+k^{r-2} b_{3} s^{2}\right. \\
& \left.+k^{r-1} b_{2} s+k^{r} b_{1}\right] \\
\tilde{p}_{k}(s) \triangleq & p(s) s^{r}+k b_{r} p(s) s^{r-1}+\cdots+k^{r} b_{1} p(s) \\
& +k^{r+1} \beta z(s) \hat{z}(s) .
\end{aligned}
$$

For implementation, it is desirable that the controller $\hat{G}_{k}(s)$ be stable. The following result characterizes controllers that are stable for all $k>0$.

Proposition 5.1: The controller $\hat{G}_{k}(s)$ given by (5.1) is asymptotically stable for all $k>0$ if and only if $\hat{b}(s) \triangleq s^{r}+b_{r} s^{r-1}+b_{r-1} s^{r-2}+\cdots+b_{2} s+b_{1}$ is Hurwitz.

Proof: Let $\lambda_{1}, \ldots, \lambda_{r}$ denote the roots of $\hat{b}(s)$. It follows that the poles of $\hat{G}_{k}(s)$ are given by $k \lambda_{1}, \ldots, k \lambda_{r}$. Therefore, for all $k>0, \hat{G}_{k}(s)$ is asymptotically stable if and only if $\hat{b}(s)$ is Hurwitz. 
The following generalization of root locus analysis can be viewed as an iterative application of Lemma 4.1.

Lemma 5.1: Let $\mu$ be a positive integer and let $c_{0}>0$. Then there exists a degree- $\mu$ monic polynomial

$$
c(s) \triangleq s^{\mu}+c_{\mu-1} s^{\mu-1}+\cdots+c_{1} s+\eta c_{0}
$$

such that $c(s)$ is Hurwitz for all $\eta \in(0,1]$. Now, assume that $c(s)$ given by (5.5) is Hurwitz for all $\eta \in(0,1]$. Furthermore, let $\nu$ be a nonnegative integer, and, for all $i=0,1, \ldots, \mu$, let $q_{i}(s)$ be a monic polynomial of degree $\nu+i$, where $q_{0}(s)$ is Hurwitz. Then, for all $\eta \in(0,1]$, the degree $\nu+\mu$ monic polynomial

$$
\begin{aligned}
\tilde{q}_{k}(s) \triangleq q_{\mu}(s)+ & k c_{\mu-1} q_{\mu-1}(s)+k^{2} c_{\mu-2} q_{\mu-2}(s) \\
& +k^{3} c_{\mu-3} q_{\mu-3}(s)+\cdots+k^{\mu} \eta c_{0} q_{0}(s)
\end{aligned}
$$

is high-gain Hurwitz. Furthermore, as $k \rightarrow \infty$, $\nu$ roots of $\tilde{q}_{k}(s)$ converge to the roots of $q_{0}(s)$, and the real parts of the remaining $\mu$ roots approach $-\infty$.

Proof: First, we show that there exists a polynomial $c(s)$ of the form (5.5) that is Hurwitz for all $\eta \in(0,1]$. Let $\hat{c}(s)=$ $(s+\sigma)^{\mu}$, where $\sigma=c_{0}^{1 / \mu}$ is the positive $\mu$ th root of $c_{0}$. Define $c(s) \triangleq \hat{c}(s)-(1-\eta) c_{0}$. For $\eta=1, c(s)$ has $\mu$ roots at $-\sigma$. It follows from root locus analysis that, for $\eta \in(0,1)$, the roots of $c(s)$ lie on concentric circles centered at $-\sigma$. Furthermore, the radii of these circles varies from 0 for $\eta=1$ to $\sigma$ for $\eta=0$. Thus, for all $\eta \in(0,1], c(s)$ is Hurwitz.

Now, write $q_{0}(s)=s^{\nu}+a_{\nu-1} s^{\nu-1}+\cdots+a_{1} s+a_{0}$. The Hurwitz conditions for the stability of $\tilde{q}_{k}(s)$ are polynomials in $k$. For sufficiently large $k$, the Hurwitz conditions for $\tilde{q}_{k}(s)$ are satisfied if and only if

$$
\begin{aligned}
& \Lambda_{1} \triangleq k c_{\mu-1}>0 \\
& \Lambda_{2} \triangleq\left|\begin{array}{cc}
k c_{\mu-1} & k^{3} c_{\mu-3} \\
1 & k^{2} c_{\mu-2}
\end{array}\right|>0 \\
& \Lambda_{3} \triangleq\left|\begin{array}{ccc}
k c_{\mu-1} & k^{3} c_{\mu-3} & k^{5} c_{\mu-5} \\
1 & k^{2} c_{\mu-2} & k^{4} c_{\mu-4} \\
0 & k c_{\mu-1} & k^{3} c_{\mu-3}
\end{array}\right|>0
\end{aligned}
$$

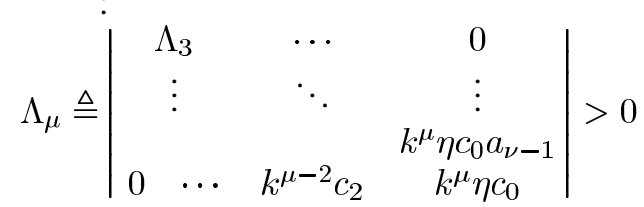

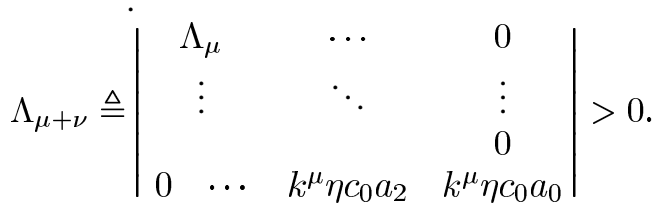

For all $k>0$ and for $j=1, \ldots, \mu, \Lambda_{j}>0$ if and only if $\hat{\Lambda}_{j} \triangleq \Lambda_{j} / k \sum_{i=1}^{j} i>0$. The conditions $\hat{\Lambda}_{1}>0, \ldots, \hat{\Lambda}_{\mu}>0$ are equivalent to the Hurwitz conditions for $c(s)$. Since $c_{0}, \ldots, c_{\mu}>0$, the last $\nu$ conditions are equivalent to the Hurwitz conditions for $q_{0}(s)$. Therefore, $\tilde{q}_{k}(s)$ is high-gain Hurwitz.
The last statement of Lemma 5.1 follows from factoring (5.6) as

$$
\begin{array}{r}
\tilde{q}_{k}(s)=q_{\mu}(s)+k c_{\mu-1}\left[q_{\mu-1}(s)+k c_{\mu-2}\left[q_{\mu-2}(s)\right.\right. \\
\left.\left.+\cdots+k c_{1}\left[q_{1}(s)+k \eta c_{0} q_{0}(s)\right]\right]\right] .
\end{array}
$$

Iteratively, applying root locus techniques to a sequence of $\mu$ relative-degree-one polynomials yields the asymptotic result. $\square$

The following result is an immediate consequence of Lemma 5.1 with $\mu=r+1, \nu=n-1, q_{0}(s)=z(s) \hat{z}(s), c_{i}=b_{i}$ for $i=0, \ldots, r$, and $q_{i}(s)=p(s) s^{i-1}$ for $i=1, \ldots, r+1$.

Theorem 5.1: Consider the closed-loop transfer function (5.2)-(5.4), and assume that the $\hat{z}(s)$ and

$$
b(s) \triangleq s^{r+1}+b_{r} s^{r}+b_{r-1} s^{r-1}+\cdots+b_{1} s+\eta b_{0}
$$

are Hurwitz for all $\eta \in(0,1]$. Then $\tilde{p}_{k}(s)$ is high-gain Hurwitz, and thus $\tilde{G}_{k}(s)$ is high-gain stable. Furthermore, as $k \rightarrow \infty$, $n-1$ roots of $\tilde{p}_{k}(s)$ converge to the roots of $z(s) \hat{z}(s)$, and the real parts of the remaining $r+1$ roots of $\tilde{p}_{k}(s)$ approach $-\infty$.

Now we comment on the robustness of the compensator (5.1) to errors in the relative degree of $G(s)$. Consider (5.1) designed for $r=2$ and satisfying the assumptions of Theorem 5.1. The closed-loop system can be shown to be high-gain stable if the actual relative degree of $G(s)$ is 0,1 , or 2 . The proof uses the same reasoning as the proof of Lemma 5.1.

Next, consider (5.1) designed for $r=3$ and satisfying the assumptions of Theorem 5.1. For example, consider the highgain-stabilizing parameter-dependent dynamic compensator

$$
\hat{G}_{k}(s)=\frac{\delta k^{4}\left(s^{2}+15 s+50\right)}{s^{3}+14 k s^{2}+71 k^{2} s+154 k^{3}} .
$$

Theorem 5.1 states that (5.9) is high-gain stabilizing for relative-degree-3 plants satisfying assumptions (A1)-(A5), where the bound on the magnitude of the high-frequency gain is $b_{0}=$ 120. Now assume that the true plant is $G(s)=60 / s^{2}$, which has relative degree 2. Fig. 2 shows that all of the closed-loop poles begin at the origin for $k=0$. As $k \rightarrow \infty$, two of the closed-loop poles converge to the stable roots of $s^{2}+15 s+50$, while one closed-loop pole approach minus infinity. However, the remaining two poles diverge to infinity through the right half plane. Thus, this controller is not high-gain stabilizing for the relative-degree-2 double integrator. In fact, the following result shows that the controller (5.1) with $r=3$ is never high-gain stabilizing for plants with relative degree 2 .

Proposition 5.2: Consider the controller (5.1) with $r=3$. If the relative degree of $G(s)$ is 2 , then $\tilde{G}_{k}(s)$ is not high-gain stable.

Proof: The Hurwitz conditions for the stability of $\tilde{p}_{k}(s)$ are polynomials in $k$. For sufficiently large $k$, the Hurwitz conditions for $\tilde{p}_{k}(s)$ are satisfied if and only if $\Lambda_{1} \triangleq k b_{3}>0, \Lambda_{2} \triangleq$ $\left|\begin{array}{cc}k b_{3} & k^{4} b_{0} \\ 1 & k^{2} b_{2}\end{array}\right|>0, \ldots$ The second condition is violated for all sufficiently large $k$. Therefore, $\tilde{p}_{k}(s)$ is not high-gain Hurwitz, and $\tilde{G}_{k}(s)$ is not high-gain stable.

Motivated by Proposition 5.2, we consider an alternative controller structure that is robust to errors in relative degree when the relative degree is greater than two. 


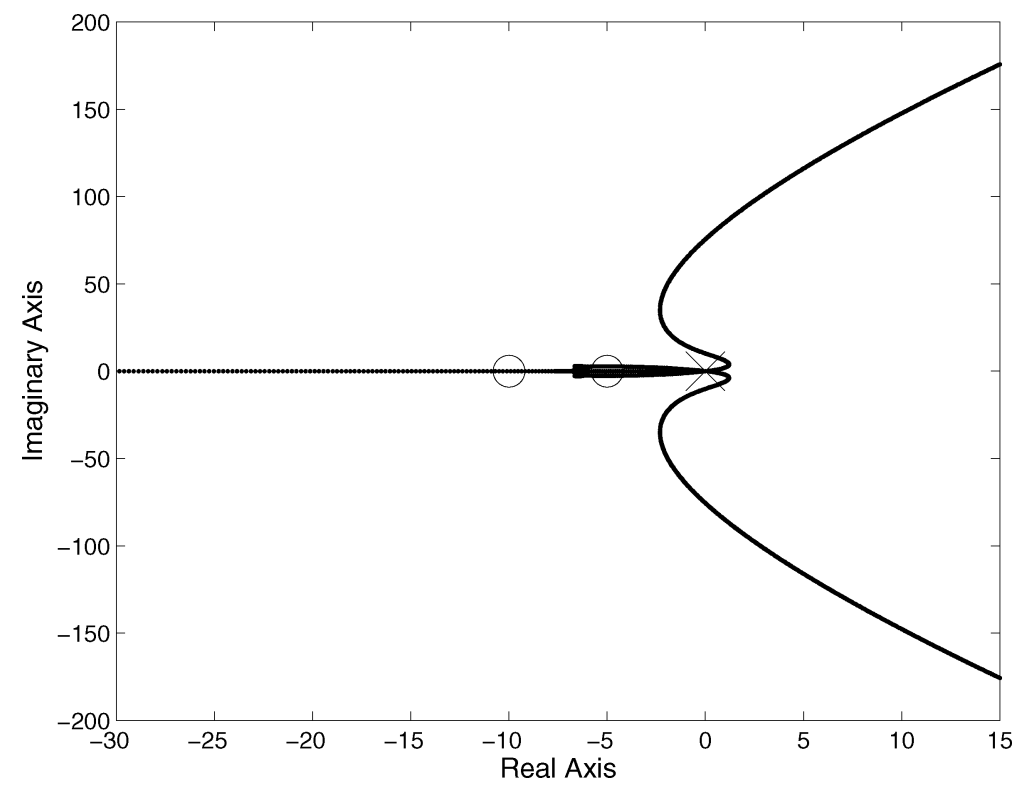

Fig. 2. Root locus for the closed-loop dynamics of the relative degree 2 plant $G(s)=60 / s^{2}$ and the controller (5.9). The system is not high-gain stable.

\section{DYNAMIC COMPENSATION FOR SYSTEMS WITH UNKNOWN-BUT-BOUNDED RELATIVE DEGREE}

We now assume that an integral bound $\rho \geq 2$ on the relative degree of (3.1) and (3.2) is known. Hence $1 \leq r \leq \rho$. For all $j \geq 0$ let $F_{j}$ be the $j$ th Fibonacci number, where $F_{0}=0$, $F_{1}=1, F_{2}=1, F_{3}=2, F_{4}=3, F_{5}=5, F_{6}=8, F_{7}=13, F_{8}=$ $21, \ldots$, and define $f_{h} \triangleq F_{\rho+2}-F_{h+1}$, where $h$ satisfies $1 \leq$ $h \leq \rho$. We use the Fibonacci numbers to construct a parameterdependent dynamic compensator, where, for sufficiently large values of the parameter $k$, the Hurwitz stability conditions on the closed-loop dynamics are satisfied and independent of $k$. The exact Fibonacci series is required in the controller construction.

Consider the feedback (3.3) with the strictly proper controller

$$
\hat{G}_{k}(s) \triangleq \frac{\delta k^{F_{\rho+2}} \hat{z}(s)}{s^{\rho}+k^{f_{\rho}} b_{\rho} s^{\rho-1}+\cdots+k^{f_{2}} b_{2} s+k^{f_{1}} b_{1}}
$$

where $k, b_{1}, \ldots, b_{\rho}$ are real and $\hat{z}(s)$ is a degree $\rho-1$ monic polynomial. The closed-loop transfer function is

$$
\tilde{G}_{k}(s) \triangleq \frac{G(s)}{1+\hat{G}_{k}(s) G(s)}=\frac{\tilde{z}_{k}(s)}{\tilde{p}_{k}(s)}
$$

where

$$
\begin{aligned}
\tilde{z}_{k}(s) \triangleq & \delta \beta z(s)\left[s^{\rho}+k^{f_{\rho}} b_{\rho} s^{\rho-1}+k^{f_{\rho-1}} b_{\rho-1} s^{\rho-2}\right. \\
& \left.+\cdots+k^{f_{2}} b_{2} s+k^{f_{1}} b_{1}\right] \\
\tilde{p}_{k}(s) \triangleq & p(s) s^{\rho}+k^{f_{\rho}} b_{\rho} p(s) s^{\rho-1}+k^{f_{\rho-1}} b_{\rho-1} p(s) s^{\rho-2} \\
& +\cdots+k^{f_{1}} b_{1} p(s)+k^{F_{\rho+2}} \beta z(s) \hat{z}(s) .
\end{aligned}
$$

The following result can be viewed as a robust version of Lemma 5.1.

Lemma 6.1: Let $\rho \geq 2$ and let $c_{\rho+1}=1$. For all $c_{0}>0$, there exist $c_{1}, \ldots, c_{\rho} \in \mathbb{R}$ such that, for all $i=0,1, \ldots, \rho-2$, the polynomials

$$
C_{i}(s) \triangleq c_{i+3} s^{3}+c_{i+2} s^{2}+c_{i+1} s+c_{0}
$$

are Hurwitz. Now, assume that $C_{0}(s), \ldots, C_{\rho-2}(s)$ given by (6.5) are Hurwitz. Furthermore, let $\nu$ be a positive integer, and, for all $i=1,2, \ldots, \rho+1$, let $q_{i}(s)$ be a monic polynomial of degree $\nu-1+i$. Finally, let $0 \leq j \leq \rho$ and let $q_{0}(s)$ be a monic Hurwitz polynomial of degree $\nu-1+j$. Then, for all $\eta \in(0,1]$, the degree $\nu+\rho$ monic polynomial

$$
\begin{aligned}
\tilde{q}_{k}(s) \triangleq & q_{\rho+1}(s)+k^{f_{\rho}} c_{\rho} q_{\rho}(s)+k^{f_{\rho-1}} c_{\rho-1} q_{\rho-1}(s) \\
& +k^{f_{\rho-2}} c_{\rho-2} q_{\rho-2}(s)+\cdots+k^{f_{2}} c_{2} q_{2}(s) \\
& +k^{f_{1}} c_{1} q_{1}(s)+k^{F_{\rho+2}} \eta c_{0} q_{0}(s)
\end{aligned}
$$

is high-gain Hurwitz. Furthermore, as $k \rightarrow \infty, \nu-1+j$ roots of $\tilde{q}_{k}(s)$ converge to the roots of $q_{0}(s)$, and the real parts of the remaining $\rho+1-j$ roots of $\tilde{q}_{k}(s)$ approach $-\infty$.

Proof: First, we show that there exist coefficients $c_{1}, \ldots, c_{\rho}$ such that the polynomials $C_{0}(s), \ldots, C_{\rho-2}(s)$ are Hurwitz. First, let $c_{\rho}>0$ and $c_{\rho-1}>0$ be such that $c_{\rho-1} c_{\rho}>c_{0} c_{\rho+1}=c_{0}$, which implies that $C_{\rho-2}(s)$ is Hurwitz. Next, let $c_{\rho-2}>c_{0} c_{\rho} / c_{\rho-1}$, which implies that $C_{\rho-3}(s)$ is Hurwitz. In the same manner, for $i=4,5, \ldots, \rho$, let $c_{\rho-i+1}>c_{0} c_{\rho-i+3} / c_{\rho-i+2}$ so that $C_{\rho-i}(s)$ is Hurwitz. Thus, $C_{0}(s), \ldots, C_{\rho-2}(s)$ are Hurwitz.

Now, suppose $j=0$ and write $q_{0}(s)=s^{\nu-1}+a_{\nu-2} s^{\nu-2}+$ $\cdots+a_{1} s+a_{0}$. The Hurwitz conditions for the stability of $\tilde{q}_{k}(s)$ are polynomials in $k$. For sufficiently large $k$, the Hurwitz conditions for $\tilde{q}_{k}(s)$ are satisfied if and only if

$$
\begin{aligned}
& \Lambda_{1} \triangleq k^{f_{\rho}} c_{\rho}>0 \\
& \Lambda_{2} \triangleq\left|\begin{array}{cc}
k^{f_{\rho}} c_{\rho} & k^{f_{\rho-2}} c_{\rho-2} \\
1 & k^{f_{\rho-1}} c_{\rho-1}
\end{array}\right|>0 \\
& \Lambda_{3} \triangleq\left|\begin{array}{ccc}
k^{f_{\rho}} c_{\rho} & k^{f_{\rho-2}} c_{\rho-2} & k^{f_{\rho-4}} c_{\rho-4} \\
1 & k^{f_{\rho-1}} c_{\rho-1} & k^{f_{\rho-3}} c_{\rho-3} \\
0 & k^{f_{\rho}} c_{\rho} & k^{f_{\rho-2}} c_{\rho-2}
\end{array}\right|>0
\end{aligned}
$$




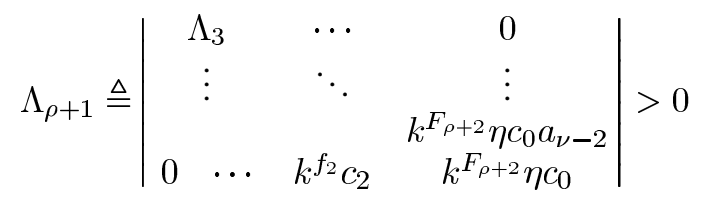

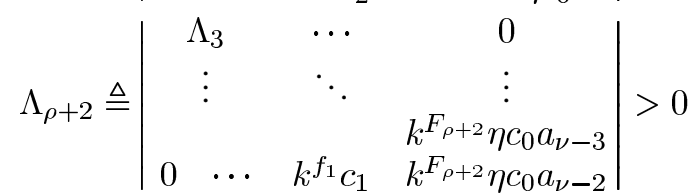

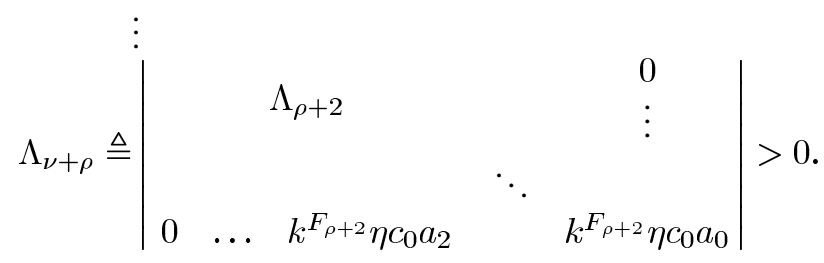

For all $k>0$, and for $i=1, \ldots, \rho, \Lambda_{i}>0$ if and only if $\hat{\Lambda}_{i} \triangleq \Lambda_{i} / k^{\sum_{j=1}^{i} f_{\rho+1-j}}>0$. Similarly, $\Lambda_{\rho+1}>0$ if and only if $\hat{\Lambda}_{\rho+1} \triangleq \Lambda_{\rho+1} /\left(k^{F_{\rho+2}+\sum_{j=1}^{\rho} f_{\rho+1-j}}\right)>0$. Since the powers of $k$ in $\Lambda_{1}, \ldots, \Lambda_{\rho+1}$ are determined by the Fibonacci series, it follows that $\hat{\Lambda}_{1}, \ldots, \hat{\Lambda}_{\rho+1}$ are independent of $k$. Furthermore, $\hat{\Lambda}_{1}>0, \ldots, \hat{\Lambda}_{\rho+1}>0$ are equivalent to $c_{i}>0$ for $i=0,1, \ldots, \rho$, and $c_{2} c_{1}-\eta c_{3} c_{0}>0$, which is satisfied since $C_{0}(s)$ is Hurwitz. Since $c_{0}, \ldots, c_{\rho}>0$, the last $\nu-1$ conditions are equivalent to the Hurwitz conditions for $q_{0}(s)$. Therefore, $\tilde{q}_{k}(s)$ is high-gain Hurwitz.

Suppose $j=1$ and write $q_{0}(s)=s^{\nu}+a_{\nu-1} s^{\nu-1}+\cdots+$ $a_{1} s+a_{0}$. For sufficiently large $k$, the Hurwitz conditions for $\tilde{q}_{k}(s)$ are satisfied if and only if

$$
\begin{aligned}
& \Lambda_{1} \triangleq k^{f_{\rho}} c_{\rho}>0 \\
& \Lambda_{2} \triangleq\left|\begin{array}{cc}
k^{f_{\rho}} c_{\rho} & k^{f_{\rho-2}} c_{\rho-2} \\
1 & k^{f_{\rho-1}} c_{\rho-1}
\end{array}\right|>0
\end{aligned}
$$

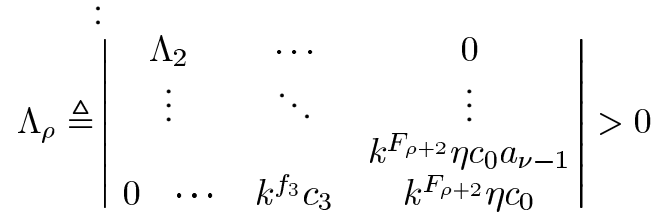

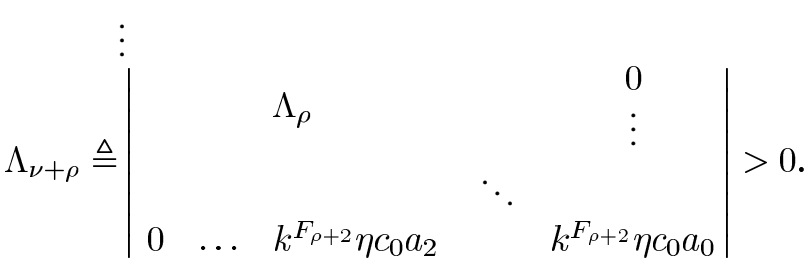

The first $\rho$ conditions are equivalent to $c_{i}>0$ for all $i=$ $0,2,3, \ldots, \rho$, and $c_{3} c_{2}-\eta c_{4} c_{0}>0$, which is satisfied since $C_{1}(s)$ is Hurwitz. Since $c_{0}, \ldots, c_{\rho}>0$, the last $\nu$ conditions are equivalent to the Hurwitz conditions for $q_{0}(s)$. Therefore, $\tilde{q}_{k}(s)$ is high-gain Hurwitz.

Suppose $j=2$ and let $q_{0}(s)$ be a degree $\nu+1$ polynomial. For sufficiently large $k$, the first $\rho-1$ Hurwitz conditions for $\tilde{q}_{k}(s)$ are equivalent to $c_{i}>0$ for $i=0,3,4, \ldots, \rho$, and $c_{4} c_{3}-\eta c_{5} c_{0}>0$, which is satisfied since $C_{2}(s)$ is Hurwitz. Since $c_{0}, \ldots, c_{\rho}>0$, the last $\nu+1$ conditions are equivalent to the Hurwitz conditions for $q_{0}(s)$. Therefore, $\tilde{q}_{k}(s)$ is high-gain Hurwitz. The same argument holds for $\operatorname{deg} q_{0}(s)=$ $\nu+2, \ldots, \nu+\rho-3$.

Suppose $j=\rho-1$ and let $q_{0}(s)$ be a degree $\nu+\rho-2$ polynomial. For sufficiently large $k$, the first two Hurwitz conditions for $\tilde{q}_{k}(s)$ are satisfied if and only if $c_{0}>0$ and $c_{\rho}>0$. The Hurwitz assumption for $q_{0}(s)$ implies that the remaining $\nu+\rho-2$ Hurwitz conditions for $\tilde{q}_{k}(s)$ are satisfied for sufficiently large $k$. Therefore, $\tilde{q}_{k}(s)$ is high-gain Hurwitz.

Suppose $j=\rho$ and let $q_{0}(s)$ be a degree $\nu+\rho-1$ polynomial. For sufficiently large $k$, the first Hurwitz condition for $\tilde{q}_{k}(s)$ is satisfied if and only if $c_{0}>0$. The Hurwitz assumption for $q_{0}(s)$ implies that the remaining $\nu+\rho-1$ Hurwitz conditions for $\tilde{q}_{k}(s)$ are satisfied for sufficiently large $k$. Therefore, $\tilde{q}_{k}(s)$ is high-gain Hurwitz.

The last statement of Lemma 5.1 follows from factoring (6.6) in $k$ in a similar fashion to (5.7). Iteratively, applying root locus techniques to a sequence of $\rho+1$ relative-degree-one polynomials yields the asymptotic result.

The following result is an immediate consequence of Lemma 6.1 with $\nu=n, j=\rho-r, q_{0}(s)=z(s) \hat{z}(s), c_{i}=b_{i}$ for $i=0, \ldots, \rho$, and $q_{i}(s)=p(s) s^{i-1}$ for $i=1, \ldots, \rho+1$.

Theorem 6.1: Consider the closed-loop transfer function (6.2)-(6.4). Assume that the polynomials $\hat{z}(s)$,

$$
B_{\rho-2}(s) \triangleq s^{3}+b_{\rho} s^{2}+b_{\rho-1} s+b_{0}
$$

and, for $i=0,1, \ldots, \rho-3$,

$$
B_{i}(s) \triangleq b_{i+3} s^{3}+b_{i+2} s^{2}+b_{i+1} s+b_{0}
$$

are Hurwitz. Then, $\tilde{p}_{k}(s)$ is high-gain Hurwitz, and thus $\tilde{G}_{k}(s)$ is high-gain stable. Furthermore, as $k \rightarrow \infty, m+\rho-1$ roots of $\tilde{p}_{k}(s)$ converge to the roots of $z(s) \hat{z}(s)$, and the real parts of the remaining $r+1$ roots approach $-\infty$.

\section{PARAMETER-Monotonic Adaptive Stabilization}

In Section VI, we presented the strictly proper compensator (6.1), where the stabilizing threshold $k_{\mathrm{s}}$ is unknown. In this section, we consider parameter-monotonic adaptive stabilization for a class of high-gain-stable systems. Although the highgain-stable closed-loop systems considered in Sections IV-VI are single-output systems, in this section we consider multipleoutput high-gain-stable systems. The following result concerns parameter-monotonic adaptive stabilization. Let $\|\cdot\|$ denote the Euclidean norm.

Theorem 7.1: Let $A(k) \in \mathbb{R}^{l \times l}$ and $C(k) \in \mathbb{R}^{d \times l}$ have polynomial entries in $k$, and assume there exists $k_{d} \in \mathbb{R}$ such that, for all $k>k_{d},(A(k), C(k))$ is detectable. Assume there exists $k_{\mathrm{s}}>0$ such that, for all $k \geq k_{\mathrm{s}}, A(k)$ is asymptotically stable. Consider the system

$$
\begin{aligned}
\dot{x}(t) & =A(k(t)) x(t) \\
y(t) & =C(k(t)) x(t)
\end{aligned}
$$

and the parameter-monotonic adaptive law

$$
\dot{k}(t)=\gamma e^{-\alpha k(t)}\|y(t)\|^{2}
$$


where $\gamma>0$ and $\alpha>0$. Then, for all initial conditions $x(0)$ and $k(0)>k_{d}, k_{\infty} \triangleq \lim _{t \rightarrow \infty} k(t)$ exists and $\lim _{t \rightarrow \infty} x(t)=0$.

Proof: Since (7.1)-(7.3) is locally Lipschitz, it follows that the solution to (7.1)-(7.3) exists and is unique locally, that is, there exists $t_{e}>0$ such that $(x(t), k(t))$ exists and is unique on the interval $\left[0, t_{e}\right)$.

First, we show that if $x(t)$ escapes at $t_{e}$, then $k(t)$ escapes at $t_{e}$. Assume that $k(t)$ does not escape at $t_{e}$. Let $k\left(t_{e}\right) \triangleq$ $\lim _{t \rightarrow t_{e}} k(t)$ so that $k(t)$ is continuous on $\left[0, t_{e}\right]$. Then, $\dot{q}(t)=$ $A(k(t)) q(t)$ is a linear time-varying differential equation on $\left[0, t_{e}\right]$, where $A(k(t))$ is continuous in $t$. The solution to $\dot{q}(t)=$ $A(k(t)) q(t)$ exists and is unique on $\left[0, t_{e}\right]$ [23]. Therefore, $q(t)$ does not escape at $t_{e}$ and, by uniqueness, $x(t)=q(t)$ on $\left[0, t_{e}\right]$. Consequently, $x(t)$ does not escape at $t_{e}$. Hence, if $x(t)$ escapes at $t_{e}$, then $k(t)$ escapes at $t_{e}$.

Now, we show that $k(t)$ converges. For contradiction, suppose that $k(t)$ escapes at $t_{e}$. Let $Q(k) \triangleq I+\gamma C^{\mathrm{T}}(k) C(k)$, and using Lemma A.1, for all $k \geq k_{\mathrm{s}}$, let $P(k)$ satisfy (A.1). Lemma A. 2 implies that there exists $k_{2} \geq k_{\mathrm{s}}$ such that, for all $k \geq k_{2}$, $\alpha P(k)>\partial P(k) / \partial k$. Since $k(t)$ diverges to infinity at $t_{e}$, there exists $t_{2}<t_{e}$ such that $k\left(t_{2}\right)=k_{2}$.

For all $t \in\left[t_{2}, t_{e}\right)$, define $V_{0}(x, k) \triangleq e^{-\alpha k} x^{\mathrm{T}} P(k) x$. Note that $V_{0}(x, k)$ is not to be considered a candidate Lyapunov function but is nonnegative for all $k \geq k_{\mathrm{s}}$ and for all $x \in \mathbb{R}^{l}$. For all $t \in\left[t_{2}, t_{e}\right)$ the derivative of $V_{0}(x, k)$ along trajectories of (7.1)-(7.3) satisfies

$$
\begin{aligned}
\dot{V}_{0}(x, k)= & -e^{-\alpha k} x^{\mathrm{T}}\left[A^{\mathrm{T}}(k) P(k)+P(k) A(k)\right] x \\
& -\alpha \dot{k} e^{-\alpha k} x^{\mathrm{T}} P(k) x+\dot{k} e^{-\alpha k} x^{\mathrm{T}} \frac{\partial P(k)}{\partial k} x \\
= & -e^{-\alpha k} x^{\mathrm{T}} Q(k) x \\
& -\dot{k} e^{-\alpha k} x^{\mathrm{T}}\left[\alpha P(k)-\frac{\partial P(k)}{\partial k}\right] x \\
\leq & -e^{-\alpha k} x^{\mathrm{T}} Q(k) x \\
= & -e^{-\alpha k} x^{\mathrm{T}}\left[I+\gamma C^{\mathrm{T}}(k) C(k)\right] x \\
\leq & -\gamma e^{-\alpha k}\|y\|^{2}=-\dot{k} .
\end{aligned}
$$

Integrating (7.4) from $t_{2}$ to $t<t_{e}$ and solving for $k(t)$ yields

$$
\begin{aligned}
k(t) & \leq V_{0}\left(x\left(t_{2}\right), k_{2}\right)+k_{2}-e^{-\alpha k(t)} x^{\mathrm{T}}(t) P(k(t)) x(t) \\
& \leq V_{0}\left(x\left(t_{2}\right), k_{2}\right)+k_{2}
\end{aligned}
$$

for $t \in\left[t_{2}, t_{e}\right)$. Hence, $k(t)$ is bounded on $\left[0, t_{e}\right)$, which is a contradiction. Therefore, the solution to (7.1)-(7.3) exists and is unique on all finite intervals. Then integrating (7.4) from $t_{2}$ to $t<\infty$ yields (7.5) for $t \in\left[t_{2}, \infty\right)$. Therefore, $k(t) \leq$ $V_{0}\left(x\left(t_{2}\right), k_{2}\right)+k_{2}$ is bounded. Since $k(t)$ is nondecreasing, $k_{\infty} \triangleq \lim _{t \rightarrow \infty} k(t)$ exists.

Since for all $t>0, k(t)<k_{\infty}$, it follows that

$$
\begin{aligned}
\gamma e^{-\alpha k_{\infty}} \int_{0}^{t}\|y(\tau)\|^{2} \mathrm{~d} \tau & \leq \gamma \int_{0}^{t} e^{-\alpha k(\tau)}\|y(\tau)\|^{2} \mathrm{~d} \tau \\
& =k(t)-k(0)<k_{\infty}-k(0)
\end{aligned}
$$

and, thus, $y(\cdot)$ is square integrable on $[0, \infty)$. This property will be used later.

Next, we show that $\lim _{t \rightarrow \infty} x(t)=0$. Define $A_{\infty} \triangleq A\left(k_{\infty}\right)$ and $C_{\infty} \triangleq C\left(k_{\infty}\right)$. Since $\left(A_{\infty}, C_{\infty}\right)$ is detectable, it follows that there exists $L \in \mathbb{R}^{l \times d}$ such that $A_{\mathrm{S}} \triangleq A_{\infty}+L C_{\infty}$ is asymptotically stable. Then, (7.1) can be written as

$$
\dot{x}(t)=A_{\mathrm{s}} x(t)+\Delta(t) x(t)-L y(t)
$$

where $\Delta(t) \triangleq A(k(t))-A_{\infty}+L C(k(t))-L C_{\infty}$. Note that $A_{\mathrm{s}}$ is asymptotically stable and $\Delta(t) \rightarrow 0$ as $t \rightarrow \infty$. Consider the function

$$
V(x) \triangleq x^{\mathrm{T}} P x
$$

where $A_{\mathrm{s}}^{\mathrm{T}} P+P A_{\mathrm{s}}=-2 I$ and $P=P^{\mathrm{T}}>0$. Taking the derivative of $V(x)$ along trajectories of (7.6) yields

$$
\begin{array}{r}
\dot{V}(x)=-2 x^{\mathrm{T}} x+x^{\mathrm{T}}\left[\Delta^{\mathrm{T}}(t) P+P \Delta(t)\right] x \\
-x^{\mathrm{T}} P L y-y^{\mathrm{T}} L^{\mathrm{T}} P x .
\end{array}
$$

Since $0 \leq(x+P L y)^{\mathrm{T}}(x+P L y)$, it follows that $-x^{\mathrm{T}} P L y-$ $y^{\mathrm{T}} L^{\mathrm{T}} P x \leq x^{\mathrm{T}} x+y^{\mathrm{T}} L^{\mathrm{T}} P^{2} L y$, which implies that

$$
\dot{V}(x) \leq-x^{\mathrm{T}} x+\lambda_{\max }\left(\Delta^{\mathrm{T}}(t) P+P \Delta(t)\right) x^{\mathrm{T}} x+\eta y^{\mathrm{T}} y
$$

where $\eta \triangleq \lambda_{\max }\left(L^{\mathrm{T}} P^{2} L\right)$. Since $\Delta(t) \rightarrow 0$ as $t \rightarrow \infty$, it follows that there exists $t_{0} \geq 0$ such that, for all $t \geq t_{0}$, $\lambda_{\max }\left(\Delta^{\mathrm{T}}(t) P+P \Delta(t)\right) \leq 1 / 2$. Therefore, for all $t \geq \bar{t}_{0}$

$$
\dot{V}(x(t)) \leq-\frac{1}{2}\|x(t)\|^{2}+\eta\|y(t)\|^{2} .
$$

Integrating from $t_{0}$ to $t$ yields

$$
\begin{aligned}
0 \leq V(x(t)) \leq & -\frac{1}{2} \int_{t_{0}}^{t}\|x(\tau)\|^{2} \mathrm{~d} \tau+\eta \int_{t_{0}}^{t}\|y(\tau)\|^{2} \mathrm{~d} \tau \\
& +V\left(x\left(t_{0}\right)\right) .
\end{aligned}
$$

Since $\eta>0$ and $y(\cdot)$ is square integrable, it follows from (7.7) that $x(\cdot)$ is square integrable and $V(\cdot)$ is bounded. Since $V(\cdot)$ is bounded, $x(\cdot)$ is bounded. Next, since $k(\cdot)$ converges, $A(\cdot)$ is continuous, and $x(\cdot)$ is bounded, it follows from (7.1) that $\dot{x}(\cdot)$ is bounded. Therefore, $(\mathrm{d} / \mathrm{d} t)\left(\|x(t)\|^{2}\right)=2 \dot{x}^{\mathrm{T}}(t) x(t)$ is bounded and, thus, $\|x(t)\|^{2}$ is uniformly continuous. Since $\|x(t)\|^{2}$ is uniformly continuous and $\lim _{t \rightarrow \infty} \int_{0}^{t}\|x(\tau)\|^{2} \mathrm{~d} \tau$ exists, Barbalat's lemma implies that $\lim _{t \rightarrow \infty} x(t)=0$.

Note that the parameter-monotonic adaptive law (7.3) contains the factor $e^{-\alpha k(t)}$, which decays exponentially as $k(t)$ increases. This factor helps to prevent $k(t)$ from growing unnecessarily large, that is, overshooting the stabilizing threshold $k_{\mathrm{s}}$. The proof of Theorem 7.1 requires that the parameter monotonic adaptive law (7.3) include the exponentially decaying factor $e^{-\alpha k(t)}$, where $\alpha>0$. This factor has no counterpart in [3] and [5]-[7].

\section{PARAMETER-MONOTONIC ADAPTIVE STABILIZATION FOR} SySTEMS With UNKNOWN-BUT-BOUNDED RELATIVE DEGREE

Now, we apply Theorem 7.1 to the strictly proper parameterdependent dynamic compensator (6.1), which stabilizes minimum-phase systems with unknown-but-bounded relative degree. To complete our analysis, we construct state-space real- 


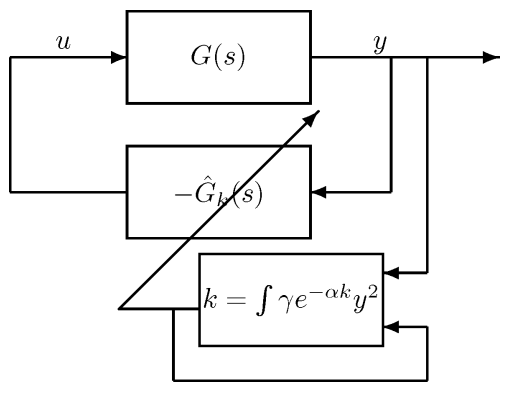

Fig. 3. Block diagram of the closed-loop adaptive system.

izations of the open-loop system (3.1) and (3.2) and the compensator (3.3) and (6.1). Let the system (3.1) and (3.2) have the minimal state-space realization

$$
\dot{x}=A x+B u \quad y=C x
$$

where $A \in \mathbb{R}^{n \times n}, B \in \mathbb{R}^{n \times 1}$, and $C \in \mathbb{R}^{1 \times n}$. This SISO plant satisfies assumptions (A1)-(A5).

Next, consider the controller $\hat{G}_{k}(s)$ given by (6.1) and write $\hat{z}(s)=s^{\rho-1}+\hat{z}_{\rho-2} s^{\rho-2}+\cdots+\hat{z}_{1} s+\hat{z}_{0}$, so that $\hat{G}_{k}(s)$ has the state-space realization

$$
\dot{\hat{x}}=\hat{A}(k) \hat{x}+\hat{B} y \quad u_{c}=\hat{C} \hat{x}
$$

where $\hat{A}(k) \in \mathbb{R}^{\rho \times \rho}, \hat{B} \in \mathbb{R}^{\rho \times 1}$, and $\hat{C}(k) \in \mathbb{R}^{1 \times \rho}$ are given by

$$
\begin{gathered}
\hat{A}(k) \triangleq\left[\begin{array}{cccc}
-k^{f_{\rho}} b_{\rho} & 1 & \cdots & 0 \\
\vdots & & \ddots & \vdots \\
-k^{f_{2}} b_{2} & 0 & & 1 \\
-k^{f_{1}} b_{1} & 0 & \cdots & 0
\end{array}\right] \quad \hat{B} \triangleq\left[\begin{array}{c}
1 \\
\hat{z}_{\rho-2} \\
\vdots \\
\hat{z}_{0}
\end{array}\right] \\
\hat{C}(k) \triangleq\left[\begin{array}{llll}
\delta k^{F_{\rho+2}} & 0 & \cdots & 0
\end{array}\right] .
\end{gathered}
$$

Note that, for all nonzero $k \in \mathbb{R},(\hat{A}(k), \hat{C}(k))$ is observable. Let $u=-u_{c}$ and the closed-loop system (8.1) and (8.2)-(8.4) is

$$
\dot{\tilde{x}}=\tilde{A}(k) \tilde{x} \quad y=\tilde{C} \tilde{x}
$$

where

$$
\tilde{A}(k) \triangleq\left[\begin{array}{cc}
A & -B \hat{C}(k) \\
\hat{B} C & \hat{A}(k)
\end{array}\right] \quad \tilde{C} \triangleq\left[\begin{array}{ll}
C & 0
\end{array}\right] \quad \tilde{x} \triangleq\left[\begin{array}{l}
x \\
\hat{x}
\end{array}\right] .
$$

Now, we present the main result of this paper. Fig. 3 illustrates the closed-loop adaptive system presented in Theorem 8.1.

Theorem 8.1: Consider the closed-loop system (8.5) and (8.6) consisting of the open-loop system (8.1) with unknown relative degree $r$ satisfying $1 \leq r \leq \rho$, and the feedback controller (8.2)-(8.4). Furthermore, consider the parameter-monotonic adaptive law

$$
\dot{k}(t)=\gamma e^{-\alpha k(t)} y^{2}(t)
$$

where $\gamma>0$ and $\alpha>0$. Assume that the polynomials $B_{0}(s), \ldots, B_{\rho-2}(s)$ given by (6.7) and (6.8) are Hurwitz, and assume that the polynomial $\hat{z}(s)$ is Hurwitz. Then, for all initial conditions $\tilde{x}(0)$ and $k(0)>0, k(t)$ converges and $\lim _{t \rightarrow \infty} \tilde{x}(t)=0$.

Proof: First, we show that for all $k>0$, the pair $(\tilde{A}(k), \tilde{C})$ is detectable. Let $\lambda$ be an element of the closed right-half plane. Then

$$
\begin{aligned}
\operatorname{rank}\left[\begin{array}{c}
\tilde{A}(k)-\lambda I \\
\tilde{C}
\end{array}\right] & =\operatorname{rank}\left[\begin{array}{cc}
A-\lambda I & -B \hat{C}(k) \\
\hat{B} C & \hat{A}(k)-\lambda I \\
C & 0
\end{array}\right] \\
& =\operatorname{rank}\left[\begin{array}{cc}
A-\lambda I & -B \hat{C}(k) \\
C & 0 \\
0 & \hat{A}(k)-\lambda I
\end{array}\right] \\
& =\operatorname{rank} \Omega\left[\begin{array}{cc}
I_{n} & 0 \\
0 & -\hat{C}(k) \\
0 & \hat{A}(k)-\lambda I
\end{array}\right]
\end{aligned}
$$

where

$$
\Omega \triangleq\left[\begin{array}{ccc}
A-\lambda I & B & 0 \\
C & 0 & 0 \\
0 & 0 & I_{\rho}
\end{array}\right]
$$

is nonsingular because $(A, B, C)$ is a minimal realization of the minimum phase plant (3.1) and (3.2). Thus, $\operatorname{rank}\left[\begin{array}{c}\tilde{A}(k)-\lambda I \\ \tilde{C}\end{array}\right]=\operatorname{rank}\left[\begin{array}{cc}I_{n} & 0 \\ 0 & -\hat{C}(k) \\ 0 & \hat{A}(k)-\lambda I\end{array}\right]$. Since, for all $k>0,(\hat{A}(k), \hat{C}(k))$ is observable, it follows that for all $k>0, \operatorname{rank}\left[\begin{array}{c}\tilde{A}(k)-\lambda I \\ \tilde{C}\end{array}\right]=n+\rho$. Therefore, for all $k>0$, $(\tilde{A}(k), \tilde{C})$ is detectable.

Theorem 6.1 implies that there exists $k_{\mathrm{s}}>0$ such that, for all $k \geq k_{\mathrm{s}}, \tilde{A}(k)$ is asymptotically stable. Since $(\tilde{A}(k), \tilde{C})$ is detectable for all $k>0$, it follows from Theorem 7.1 that $k(t)$ converges and $\lim _{t \rightarrow \infty} \tilde{x}(t)=0$.

Theorem 8.1 presents an adaptive compensator for systems with unknown-but-bounded relative degree. If the relative degree $r$ of the plant $G(s)$ is arbitrary-but-known, then the controller (5.1) can be used with the parameter-monotonic adaptive law $\dot{k}(t)=\gamma e^{-\alpha k(t)} y^{2}(t)$. The conclusions and proof remains unchanged.

Theorem 8.2: Consider the closed-loop system consisting of the open-loop system (8.1) and the feedback controller (8.2), where $u=-u_{c}$ and $(\hat{A}(k), \hat{B}, \hat{C}(k))$ is a state-space realization of (5.1) given by

$$
\begin{aligned}
& \hat{A}(k) \triangleq\left[\begin{array}{cccc}
-k b_{r} & 1 & \cdots & 0 \\
\vdots & & \ddots & \vdots \\
-k^{r-2} b_{2} & 0 & & 1 \\
-k^{r-1} b_{1} & 0 & \cdots & 0
\end{array}\right] \quad \hat{B} \triangleq\left[\begin{array}{c}
1 \\
\hat{z}_{r-2} \\
\vdots \\
\hat{z}_{0}
\end{array}\right] \\
& \hat{C}(k) \triangleq\left[\begin{array}{llll}
\delta k^{r+1} & 0 & \cdots & 0
\end{array}\right]
\end{aligned}
$$

where $\hat{z}(s)=s^{r-1}+\hat{z}_{r-2} s^{r-2}+\cdots+\hat{z}_{1} s+\hat{z}_{0}$. Let $\tilde{x}(t) d e$ note the state of the closed-loop system. Furthermore, consider the parameter-monotonic adaptive law $\dot{k}(t)=\gamma e^{-\alpha k(t)} y^{2}(t)$, where $\gamma>0$ and $\alpha>0$. Assume that, for all $\eta \in(0,1]$, the polynomial $b(s)$ given by (5.8) is Hurwitz, and assume that the polynomial $\hat{z}(s)$ is Hurwitz. Then, for all initial conditions $\tilde{x}(0)$ and $k(0)>0, k(t)$ converges and $\lim _{t \rightarrow \infty} \tilde{x}(t)=0$. 


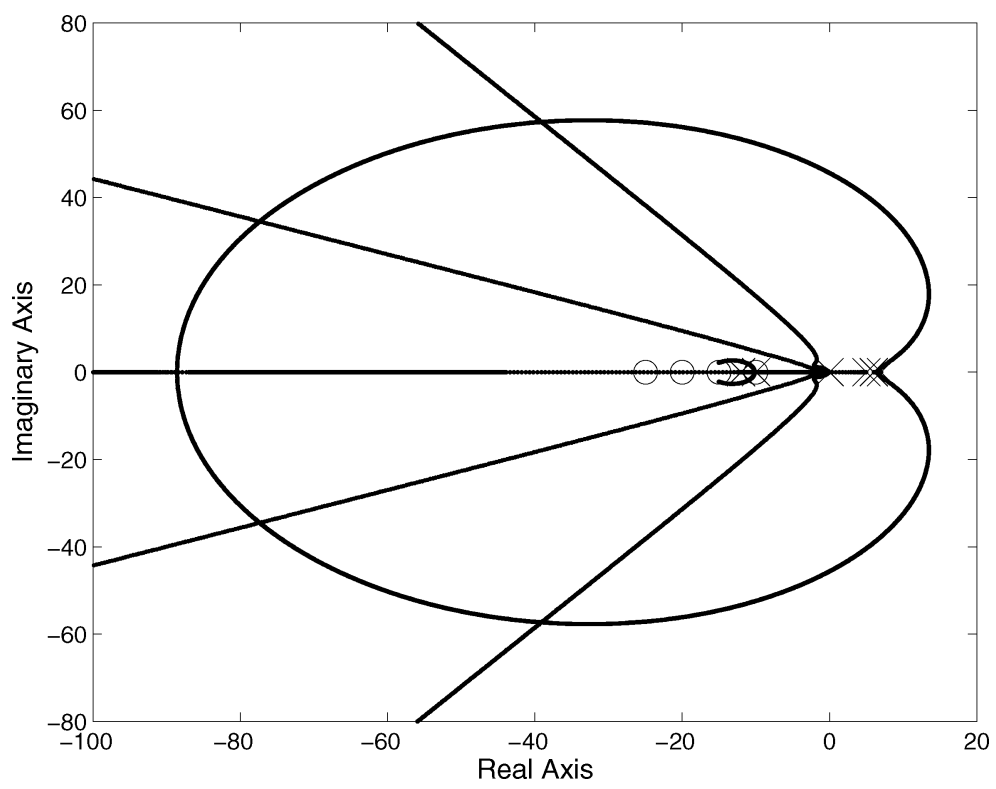

Fig. 4. Root locus of the closed-loop dynamics using parameter-dependent dynamic compensation. The closed-loop system is high-gain stable.

\section{CountereXample to the Results of [7] ReVisited}

In this section, we consider the system $y=G(s) u$ where $G(s)$ is the unstable plant (2.1). It was shown in Section II that the tenth-order controller proposed in [7] fails to stabilize (2.1). In contrast, consider the fifth-order parameter-dependent dynamic compensator

$$
\hat{G}_{k}(s)=\frac{\delta k^{6} \hat{z}(s)}{s^{5}+k b_{5} s^{4}+k^{2} b_{4} s^{3}+k^{3} b_{3} s^{2}+k^{4} b_{2} s+k^{5} b_{1}}
$$

where $\hat{z}(s)$ is a degree 4 monic Hurwitz polynomial. We assume that the high-frequency gain of (2.1) is known to be positive and that $b_{0}=2.5$ as in Section II. To satisfy the assumptions of Theorem 5.1, the numerator polynomial is chosen to be $\hat{z}(s)=$ $(s+10)(s+15)(s+20)(s+25)$ and the design parameters are chosen to be $b_{1}=13.5, b_{2}=30, b_{3}=35, b_{4}=22.5$, and $b_{5}=7.5$.

Fig. 4 illustrates the root locus for the closed-loop characteristic polynomial as $k \rightarrow \infty$. The zero-gain $(k=0)$ pole locations are shown by $\times$ 's, while the zero locations, which attract certain poles, are shown by o's. Four of the closed-loop poles converge to the stable zero locations as $k \rightarrow \infty$. The remaining six closed-loop poles diverge to infinity through the left-half plane. Thus, the closed-loop system is high-gain stable.

Theorem 8.2 yields the adaptive controller

$$
\begin{aligned}
\dot{\hat{x}} & =\left[\begin{array}{ccccc}
-7.5 & 1 & 0 & 0 & 0 \\
-22.5 k^{2} & 0 & 1 & 0 & 0 \\
-35 k^{3} & 0 & 0 & 1 & 0 \\
-30 k^{4} & 0 & 0 & 0 & 1 \\
-13.5 k^{5} & 0 & 0 & 0 & 0
\end{array}\right] \hat{x}+\left[\begin{array}{c}
1 \\
70 \\
1775 \\
19250 \\
75000
\end{array}\right] y \\
\dot{k} & =\gamma e^{-\alpha k} y^{2} \\
u & =\left[\begin{array}{lllll}
-k^{6} & 0 & 0 & 0 & 0
\end{array}\right] \hat{x}
\end{aligned}
$$

where we choose $\gamma=1000$ and $\alpha=0.01$.

The system $y=G(s) u$ is simulated with the initial condition $x(0)=\left[\begin{array}{lllll}0.1 & -0.1 & -0.5 & 1.5 & 2.0\end{array}\right]^{\mathrm{T}}$, where the state $x(\cdot)$ corresponds to a controllable canonical realization of $G(s)$. The adaptive controller (9.1)-(9.3) is implemented in the feedback loop with the initial conditions $k(0)=0$ and $\hat{x}(0)=0$. Fig. 5 shows that the output $y(t)$ of the closed-loop system converges to zero, while the adaptive parameter $k(t)$ converges to approximately 708 .

\section{Adaptive Controller for Either the Double or TRIPLE INTEGRATOR}

Consider the uncertain system $y=G(s) u$, where the sign of the high-frequency gain is positive, the upper bound on the magnitude of the high-frequency gain is $b_{0}=5$, and the bound on the relative degree is $\rho=3$. Furthermore, consider the parameter-dependent dynamic compensator

$$
\hat{G}_{k}(s)=\frac{\delta k^{5} \hat{z}(s)}{s^{3}+k^{2} b_{3} s^{2}+k^{3} b_{2} s+k^{4} b_{1}}
$$

where $\hat{z}(s)$ is a degree 2 monic Hurwitz polynomial. To satisfy the assumptions of Theorem 6.1, the design parameters are chosen to be $\hat{z}(s)=(s+10)(s+5), b_{1}=4, b_{2}=6$, and $b_{3}=4$. Theorem 8.1 yields the adaptive controller

$$
\begin{aligned}
& \dot{\hat{x}}=\left[\begin{array}{lll}
-4 k^{2} & 1 & 0 \\
-6 k^{3} & 0 & 1 \\
-4 k^{4} & 0 & 0
\end{array}\right] \hat{x}+\left[\begin{array}{c}
1 \\
15 \\
50
\end{array}\right] y \\
& \dot{k}=\gamma e^{-\alpha k} y^{2} \\
& u=\left[\begin{array}{lll}
-k^{5} & 0 & 0
\end{array}\right] \hat{x}
\end{aligned}
$$

where we choose $\gamma=100$ and $\alpha=0.1$.

Let the unknown system be the triple integrator $G(s)=2 / s^{3}$. The triple integrator is simulated with the initial condition $x(0)=\left[\begin{array}{lll}0.1 & -0.1 & -0.5\end{array}\right]^{\mathrm{T}}$, where the state $x(\cdot)$ corresponds to a controllable canonical realization of $G(s)$. The adaptive controller (10.1)-(10.3) is implemented in the feedback loop with the initial conditions $k(0)=0$ and $\hat{x}(0)=0$. Fig. 6 shows the time history of the output $y(t)$ of the closed-loop system. The output convergence rate depends on 

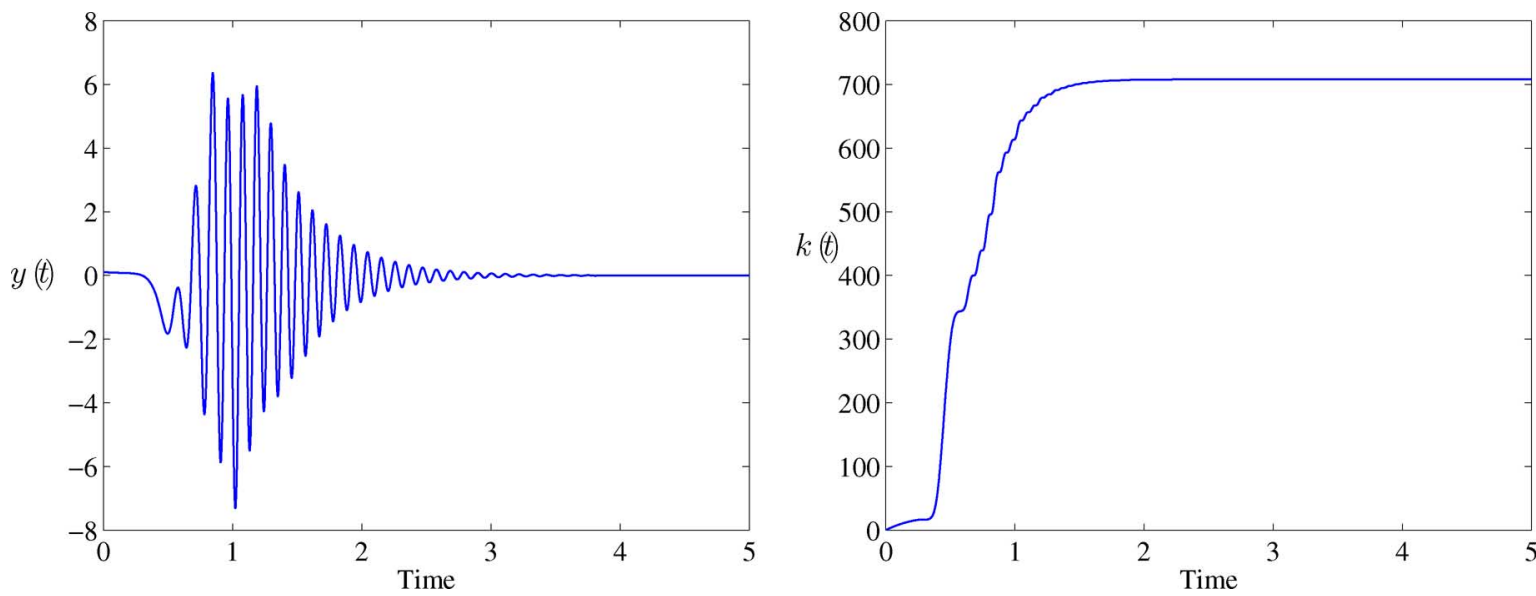

Fig. 5. Time histories of the output $y(t)$ (left) and the adaptive parameter $k(t)$ (right) for the closed-loop system consisting of the open-loop system $y=G(s) u$ and the adaptive controller $(9.1)-(9.3)$
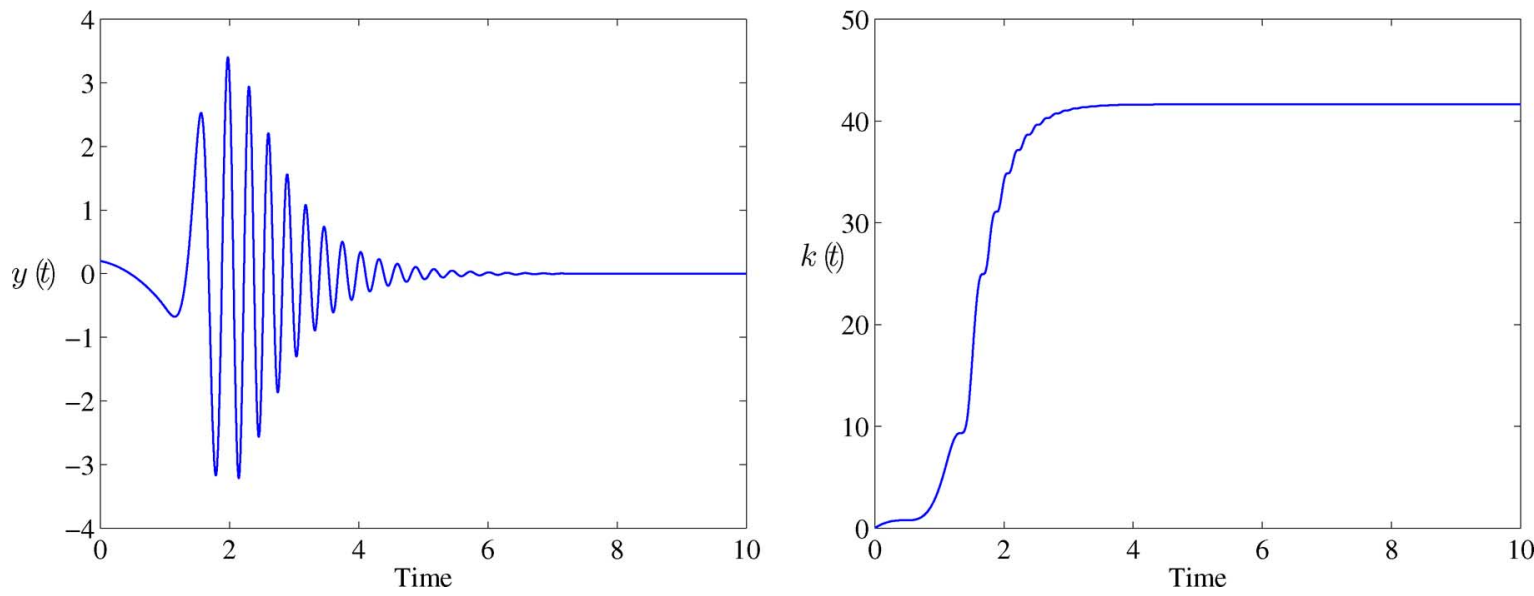

Fig. 6. Time histories of the output $y(t)$ (left) and the adaptive parameter $k(t)$ (right) for the closed-loop system consisting of the triple integrator $G(s)=2 / s^{3}$ and the adaptive controller (10.1)-(10.3).

the open-loop zeros $z(s)$ and the design parameters $b_{1}, b_{2}, b_{3}$, and $\hat{z}(s)$. In this example, there are no open-loop zeros so the choice of $\hat{z}(s)$, which effectively places two of the closed-loop poles, most directly affects the convergence rate. Fig. 6 also shows the time history of the adaptive parameter $k(t)$, which converges to approximately 41.6.

Now, let the unknown system be the double integrator $G(s)=$ $4 / s^{2}$. The double integrator is simulated with the initial condition $x(0)=\left[\begin{array}{ll}0.1 & -0.1\end{array}\right]^{\mathrm{T}}$, where the state $x(\cdot)$ corresponds to a controllable canonical realization of $G(s)$. The adaptive controller is implemented in the feedback loop with the same parameters and the initial conditions $k(0)=0$ and $\hat{x}(0)=0$. Fig. 7 shows that the output $y(t)$ converges to zero, while the adaptive parameter $k(t)$ converges to approximately 14.5.

\section{CONCLUSION}

In this paper, we presented a direct adaptive controller for SISO minimum phase linear time-invariant systems with unknown-but-bounded relative degree. The controller requires knowledge of the sign of the high-frequency gain and an upper bound on the magnitude of the high frequency gain. The adaptive controller, constructed using generalized root locus principles and the Fibonacci series, guarantees state convergence to zero. Unlike model reference adaptive control methods, this control does not require knowledge of the system order and requires only an upper bound on the relative degree. Furthermore, the adaptive controller presented herein has only one adaptive parameter regardless of system order. The order of the adaptive controller increases linearly with the relative degree of the plant. As with many parameter-monotonic adaptive laws, persistent output disturbance can cause the adaptive parameter to grow without bound. However, this problem can be corrected for in practice by turning off the adaptation mechanism once the system become stables. The adaptive controller presented in this paper has direct application to command following and disturbance rejection problems where the control objective is output convergence, see [24]. Future work includes extensions to MIMO systems.

\section{APPENDIX}

\section{PRELIMINARY RESULTS FOR ANALYZING PARAMETER-MONOTONIC ADAPTIVE SYSTEMS}

Lemma A.1: Let $A(k) \in \mathbb{R}^{l \times l}$ have entries that are polynomials in $k$. Assume that there exists $k_{\mathrm{S}}>0$ such that, for all $k \geq k_{\mathrm{s}}, A(k)$ is asymptotically stable. Let $Q(k) \in \mathbb{R}^{l \times l}$ have entries that are polynomial functions of $k$ where, for all $k \geq k_{\mathrm{s}}$, 

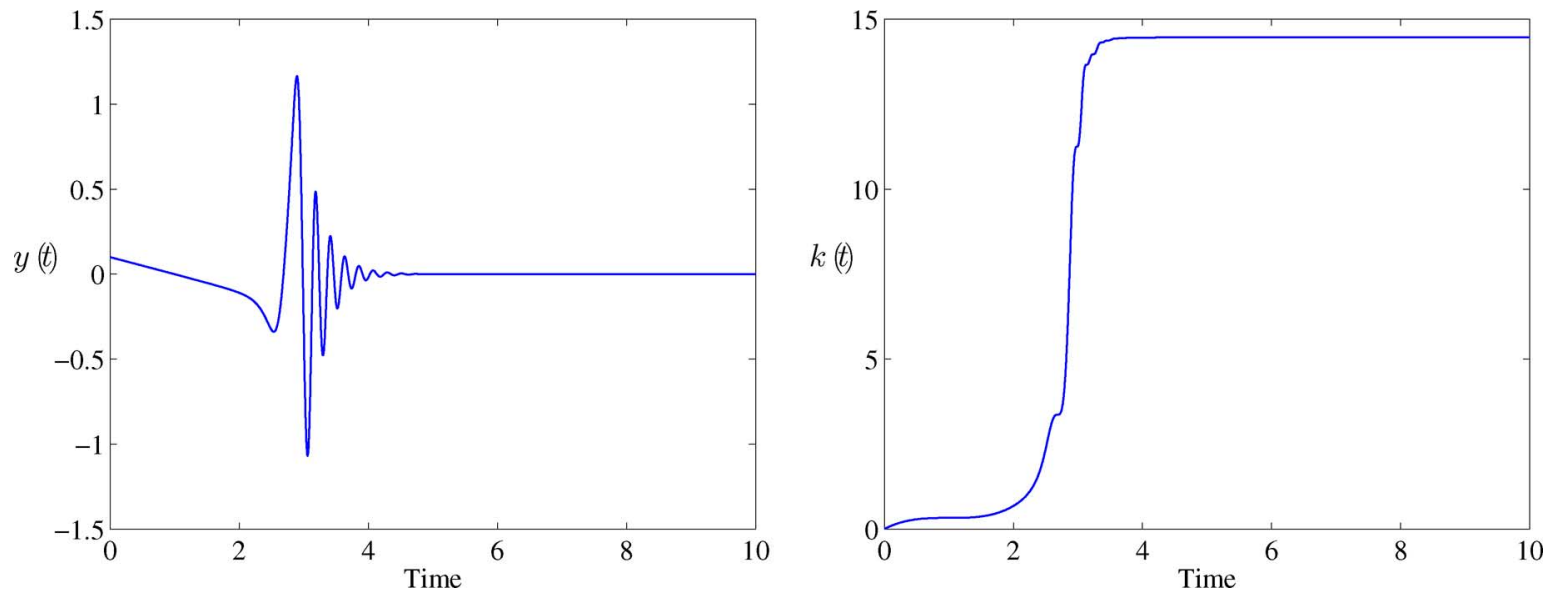

Fig. 7. Time histories of the output $y(t)$ (left) and adaptive parameter $k(t)$ (right) for the closed-loop system consisting of the double integrator $G(s)=4 / s^{2}$ and the adaptive controller $(10.1)-(10.3)$.

$Q(k)$ is positive definite. Then there exists $P:\left[k_{\mathrm{s}}, \infty\right) \rightarrow \mathbb{R}^{l \times l}$ such that each entry of $P$ is a real rational function, and for all $k \geq k_{\mathrm{s}}, P(k)$ is positive definite and satisfies

$$
A^{\mathrm{T}}(k) P(k)+P(k) A(k)=-Q(k) .
$$

Proof: For all $k \geq k_{\mathrm{s}}$, (A.1) has the unique solution

$$
\begin{aligned}
P(k) & \triangleq-\operatorname{vec}^{-1}\left[\left(A^{\mathrm{T}}(k) \oplus A^{\mathrm{T}}(k)\right)^{-1} \operatorname{vec} Q(k)\right] \\
& =\int_{0}^{\infty} e^{A^{\mathrm{T}}(k) \tau} Q(k) e^{A(k) \tau} \mathrm{d} \tau
\end{aligned}
$$

where vec is the column stacking operator and $\oplus$ is the Kronecker sum [25, Prop. 11.8.3]. Then, $P(k)$ is positive definite with entries that are real rational functions of $k$.

The entries of $P(k)$ are not necessarily proper rational functions of $k$.

We recall the $O$-notation. Let $f: \mathbb{R} \rightarrow \mathbb{R}$ and $g: \mathbb{R} \rightarrow \mathbb{R}$ be continuous functions. Then $f(k)=O(g(k))$ as $k \rightarrow \infty$ if there exist $M>0$ and $k_{1}>0$ such that, for all $k \geq k_{1}$, $|f(k)| \leq M|g(k)|$. For convenience, we will omit " $k \rightarrow \infty$ " from the $O$-notation for the remainder of this paper.

Lemma A.2: Let $k_{\mathrm{s}}>0$ and consider $P:\left[k_{\mathrm{s}}, \infty\right) \rightarrow \mathbb{R}^{l \times l}$, where each entry of $P$ is a real rational function. Assume that, for all $k \geq k_{\mathrm{s}}, P(k)$ is positive definite. Then, for all $\alpha>0$, there exists $k_{2} \geq k_{\mathrm{s}}$ such that, for all $k \geq k_{2}$

$$
\frac{\mathrm{d} P(k)}{\mathrm{d} k}<\alpha P(k)
$$

Proof: Let $P(k)=\left[\begin{array}{ccc}p_{11}(k) & \cdots & p_{1 l}(k) \\ \vdots & & \vdots \\ p_{l 1}(k) & \cdots & p_{l l}(k)\end{array}\right]$ $i, j=1, \ldots, l, p_{i j}(k)$ is the real rational function

$$
\begin{aligned}
p_{i j}(k) & \triangleq \frac{\varphi_{i j}(k)}{\psi_{i j}(k)} \\
& =\frac{\varphi_{i j, u_{i j}} k^{u_{i j}}+\varphi_{i j, u_{i j}-1} k^{u_{i j}-1}+\cdots+\varphi_{i j, 1} k+\varphi_{i j, 0}}{k^{v_{i j}}+\psi_{i j, v_{i j}-1} k^{v_{i j}-1}+\cdots+\psi_{i j, 1} k+\psi_{i j, 0}}
\end{aligned}
$$

where $u_{i j}$ and $v_{i j}$ are nonnegative integers. Then, for all $k>0$, $p_{i j}(k)$ can be expressed as

$$
p_{i j}(k)=\phi_{i j} k^{\sigma_{i j}}+\frac{k^{v_{i j}} \varphi_{i j}(k)-\phi_{i j} k^{u_{i j}} \psi_{i j}(k)}{k^{v_{i j}} \psi_{i j}(k)}
$$

where $\sigma_{i j} \triangleq u_{i j}-v_{i j}$ is an integer and $\phi_{i j} \triangleq \varphi_{i j, u_{i j}}$. The term $\left(k^{v_{i j}} \varphi_{i j}(k)-\phi_{i j} k^{u_{i j}} \psi_{i j}(k)\right) / k^{v_{i j}} \psi_{i j}(k)$ is a real rational function of $k$ whose denominator is a polynomial in $k$ of degree $2 v_{i j}$ and whose numerator is a polynomial in $k$ of degree at most $u_{i j}+v_{i j}-1$. Therefore, $\left(k^{v_{i j}} \varphi_{i j}(k)-\phi_{i j} k^{u_{i j}} \psi_{i j}(k)\right) / k^{v_{i j}} \psi_{i j}(k)=O\left(k^{\sigma_{i j}-1}\right)$ and, hence, $p_{i j}(k)=\phi_{i j} k^{\sigma_{i j}}+O\left(k^{\sigma_{i j}-1}\right)$.

For all $k \geq k_{\mathrm{s}}$, the determinants of the leading principal minors of $P(k)$ are

$$
\begin{aligned}
& \Lambda_{1}(k) \triangleq p_{11}(k)=\phi_{11} k^{\sigma_{11}}+O\left(k^{\sigma_{11}-1}\right) \\
& =\Phi_{1} k^{w_{1}}+O\left(k^{w_{1}-1}\right) \\
& \Lambda_{2}(k) \triangleq\left|\begin{array}{ll}
p_{11}(k) & p_{12}(k) \\
p_{12}(k) & p_{22}(k)
\end{array}\right| \\
& =\left|\begin{array}{ll}
\phi_{11} k^{\sigma_{11}}+O\left(k^{\sigma_{11}-1}\right) & \phi_{12} k^{\sigma_{12}}+O\left(k^{\sigma_{12}-1}\right) \\
\phi_{12} k^{\sigma_{12}}+O\left(k^{\sigma_{12}-1}\right) & \phi_{22} k^{\sigma_{22}}+O\left(k^{\sigma_{22}-1}\right)
\end{array}\right| \\
& =\Phi_{2} k^{w_{2}}+O\left(k^{w_{2}-1}\right) \\
& \Lambda_{3}(k) \triangleq\left|\begin{array}{lll}
p_{11}(k) & p_{12}(k) & p_{13}(k) \\
p_{12}(k) & p_{22}(k) & p_{23}(k) \\
p_{13}(k) & p_{23}(k) & p_{33}(k)
\end{array}\right| \\
& =\Phi_{3} k^{w_{3}}+O\left(k^{w_{3}-1}\right) \\
& \text { : } \\
& \Lambda_{l}(k) \triangleq|P(k)|=\Phi_{l} k^{w_{l}}+O\left(k^{w_{l}-1}\right)
\end{aligned}
$$

where, for $i=1, \ldots, l, w_{i}$ is an integer, and $\Phi_{i} \in \mathbb{R}$ depends on the constants $\phi_{11}, \ldots, \phi_{1 i}, \ldots, \phi_{i i}$. Since $P(k)$ is positive definite for all $k \geq k_{\mathrm{s}}$, it follows that, for all $k \geq k_{\mathrm{s}}$ and for $i=1, \ldots, l, \Lambda_{i}(k)>0$ and, thus, $\Phi_{i}>0$. 
Next, consider the derivative of $P(k)$ with respect to $k$. For $i, j=1, \ldots, l, \mathrm{~d} p_{i j}(k) / \mathrm{d} k=\sigma_{i j} \phi_{i j} k^{\sigma_{i j}-1}+O\left(k^{\sigma_{i j}-2}\right)$. Let $\alpha>0$. Then, for $i, j=1, \ldots, l$

$$
\begin{aligned}
\alpha p_{i j}(k)-\frac{\mathrm{d} p_{i j}(k)}{\mathrm{d} k}= & \alpha\left[\phi_{i j} k^{\sigma_{i j}}+O\left(k^{\sigma_{i j}-1}\right)\right] \\
& -\left[\sigma_{i j} \phi_{i j} k^{\sigma_{i j}-1}+O\left(k^{\sigma_{i j}-2}\right)\right] \\
= & \alpha \phi_{i j} k^{\sigma_{i j}}+O\left(k^{\sigma_{i j}-1}\right) .
\end{aligned}
$$

For all $k \geq k_{\mathrm{s}}$, the determinants of the leading principal minors of $\alpha P(k)-\mathrm{d} P(k) / \mathrm{d} k$ are

$$
\begin{aligned}
& \bar{\Lambda}_{1}(k) \triangleq \alpha p_{11}(k)-\frac{\mathrm{d} p_{11}(k)}{\mathrm{d} k}=\alpha \phi_{11} k^{\sigma_{11}}+O\left(k^{\sigma_{11}-1}\right) \\
& =\alpha \Phi_{1} k^{w_{1}}+O\left(k^{w_{1}-1}\right) \\
& \bar{\Lambda}_{2}(k) \triangleq\left|\begin{array}{ll}
\alpha p_{11}(k)-\frac{\mathrm{d} p_{11}(k)}{\mathrm{d} k} & \alpha p_{12}(k)-\frac{\mathrm{d} p_{12}(k)}{\mathrm{d} k} \\
\alpha p_{12}(k)-\frac{\mathrm{d} p_{12}(k)}{\mathrm{d} k} & \alpha p_{22}(k)-\frac{\mathrm{d} p_{22}(k)}{\mathrm{d} k}
\end{array}\right| \\
& =\left|\alpha\left[\begin{array}{ll}
\phi_{11} k^{\sigma_{11}}+O\left(k^{\sigma_{11}-1}\right) & \phi_{12} k^{\sigma_{12}}+O\left(k^{\sigma_{12}-1}\right) \\
\phi_{12} k^{\sigma_{12}}+O\left(k^{\sigma_{12}-1}\right) & \phi_{22} k^{\sigma_{22}}+O\left(k^{\sigma_{22}-1}\right)
\end{array}\right]\right| \\
& =\alpha^{2} \Phi_{2} k^{w_{2}}+O\left(k^{w_{2}-1}\right) \\
& \bar{\Lambda}_{3}(k) \triangleq\left|\begin{array}{ccc}
\alpha p_{11}(k)-\frac{\mathrm{d} p_{11}(k)}{\mathrm{d} k} & \cdots & \alpha p_{13}(k)-\frac{\mathrm{d} p_{13}(k)}{\mathrm{d} k} \\
\vdots & & \vdots \\
\alpha p_{13}(k)-\frac{\mathrm{d} p_{13}(k)}{\mathrm{d} k} & \cdots & \alpha p_{33}(k)-\frac{\mathrm{d} p_{33}(k)}{\mathrm{d} k}
\end{array}\right| \\
& =\alpha^{3} \Phi_{3} k^{w_{3}}+O\left(k^{w_{3}-1}\right) \\
& \bar{\Lambda}_{l}(k) \triangleq\left|\alpha P(k)-\frac{\mathrm{d} P(k)}{\mathrm{d} k}\right|=\alpha^{l} \Phi_{l} k^{w_{l}}+O\left(k^{w_{l}-1}\right) .
\end{aligned}
$$

Since $\alpha>0$, it follows that, for $i=1, \ldots, l, \alpha^{i} \Phi_{i}>0$. Therefore, for all sufficiently large $k, \bar{\Lambda}_{1}(k), \ldots, \bar{\Lambda}_{l}(k)>0$ and, thus, $\alpha P(k)-\mathrm{d} P(k) / \mathrm{d} k$ is positive definite.

\section{ACKNOWLEDGMENT}

The authors would like to extend their thanks to L. Rodman and S. Morse for their helpful discussions, and the reviewers who pointed out errors in an earlier version of this paper.

\section{REFERENCES}

[1] K. J.Åström and B. Wittenmark, Adaptive Control, 2nd ed. Reading, MA: Addison-Wesley, 1995.

[2] P. Ioannou and J. Sun, Robust Adaptive Control. Upper Saddle River, NJ: Prentice-Hall, 1996.

[3] A. L. Fradkov, "Synthesis of an adaptive system for linear plant stablization," Automat. Remote Control, vol. 35, pp. 1960-1966, 1974.

[4] B. Egardt, "Stability analysis of continuous-time adaptive control," SIAM J. Control Optim., vol. 18, pp. 540-558, 1980.

[5] C. I. Byrnes and J. C. Willems, "Adaptive stabilization of multivariable linear systems," in Proc. Conf. Decision Control, Las Vegas, NV, 1984, pp. 1574-1577.

[6] J. Willems and C. I. Byrnes, Global Adaptive Stabilization in the Absence of Information on the Sign of the High Frequency Gain, ser. Lecture Notes Control and Information Sciences. New York: SpringerVerlag, 1984, vol. 62, pp. 49-57.

[7] I. Mareels, "A simple selftuning controller for stably invertible systems," Syst. Control Lett., vol. 4, pp. 5-16, 1984.

[8] A. Ilchmann, Non-Identifier-Based High-Gain Adaptive Control. New York: Springer-Verlag, 1993.
[9] I. Mareels and J. W. Polderman, Adaptive Systems: An Introduction. Boston, MA: Birkhäuser, 1996.

[10] H. Kaufman, I. Barkana, and K. Sobel, Direct Adaptive Control Algorithms, Theory and Applications, 2nd ed. New York: Springer-Verlag, 1998.

[11] J. W. Polderman and I. M. Y. Mareels, "High gain adaptive control revisited: First and second order case," in Proc. Conf. Decision Control, Phoenix, AZ, 1999, pp. 3329-3333.

[12] A. Ilchmann and E. P. Ryan, "On gain adaptation in adaptive control," IEEE Trans. Autom. Control, vol. 48, no. 5, pp. 895-899, May 2003.

[13] R. D. Nussbaum, "Some remarks on a conjecture in parameter adaptive control," Syst. Control Lett., vol. 3, pp. 243-246, 1983.

[14] B. Martensson, "The order of any stabilizing regulator is sufficient a priori information for adaptive stabilization," Syst. Control Lett., vol. 6, pp. 87-91, 1985.

[15] D. E. Miller and E. J. Davison, "An adaptive controller which provides Lyapunov stability," IEEE Trans. Autom. Control, vol. 34, no. 6, pp. 599-609, Jun. 1989.

[16] A. S. Morse, "A three-dimensional universal controller for the adaptive stabilization of any strictly proper minimum phase system with relative degree not exceeding two," IEEE Trans. Autom. Control, vol. AC-30, no. 12 , pp. 1188-1191, Dec. 1985.

[17] E. P. Ryan, "A universal adaptive stabilizer for a class of nonlinear systems," Syst. Control Lett., vol. 16, pp. 209-218, 1991.

[18] A. Ilchmann, "Universal adaptive stabilization of nonlinear systems," Dyna. Control, vol. 7, pp. 199-213, 1997.

[19] Y. Xudong, "Universal $\lambda$-tracking for nonlinearly-perturbed systems without restrictions on the relative degree," Automatica, vol. 35, pp. 109-119, 1999.

[20] E. Bullinger and F. A. wer, "Adaptive $\lambda$-tracking for nonlinear higher relative degree systems," Automatica, vol. 41, pp. 1191-1200, 2005.

[21] A. G. J. MacFarlane and N. Karkanias, "Poles and zeros of linear multivariable systems," Int. J. Control, vol. 24, pp. 33-74, 1976.

[22] B. Kouvaritakis and A. G. J. MacFarlane, "Geometric approach to analysis and synthesis of system zeros-Part 1: Square systems," Int. J. Control, vol. 23, pp. 149-166, 1976.

[23] W. J. Rugh, Linear Systems Theory, 2nd ed. Upper Saddle River, NJ: Prentice-Hall, 1996.

[24] J. B. Hoagg and D. S. Bernstein, "Direct adaptive command following and disturbance rejection for minimum-phase systems with unknown relative degree," in Proc. Conf. Decision Control, Seville, Spain, Dec. 2005, pp. 2242-2247.

[25] D. S. Bernstein, Matrix Mathematics. Princeton, NJ: Princeton Univ Press, 2005.

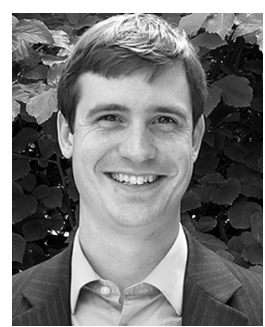

Jesse B. Hoagg received the B.S.E. degree in civil and environmental engineering from Duke University, Durham, NC, in 2002, the M.S.E degree in aerospace engineering from the University of Michigan, Ann Arbor, in 2003, the M.S. degree in mathematics from the University of Michigan in 2005, and the Ph.D. degree in aerospace engineering from the University of Michigan in 2006.

His professional experience includes appointments as a National Defense Science and Engineering Graduate Fellow for the U.S. Department of Defense and as a Space Scholar for the Space Vehicles Directorate of the U.S. Air Force Research Laboratory. His research interests are in adaptive control and control of structures.

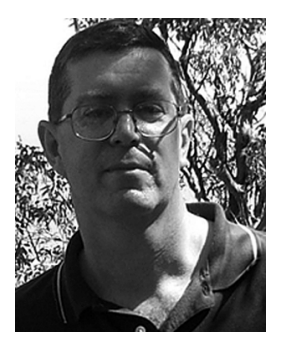

Dennis S. Bernstein received the Ph.D. degree from the University of Michigan, Ann Arbor, in 1982.

$\mathrm{He}$ is currently a Professor in the Aerospace Engineering Department of the University of Michigan. $\mathrm{He}$ is the author of Matrix Mathematics (Princeton, NJ: Princeton Univ. Press, 2005). His interests are in system identification and adaptive control for aerospace applications.

Dr. Bernstein is the Editor-in-Chief of the IEEE Control Systems Magazine. 